\title{
First Solar Orbiter observation of the Alfvénic slow wind and identification of its solar source
}

R. D’Amicis ${ }^{1}$, R. Bruno ${ }^{1}$, O. Panasenco ${ }^{2}$, D. Telloni ${ }^{3}$, D. Perrone ${ }^{4}$, M. F. Marcucci ${ }^{1}$, L. Woodham ${ }^{5}$, M. Velli ${ }^{6}$, R. De Marco $^{1}$, V. Jagarlamudi ${ }^{1}$, I. Coco ${ }^{7}$, C. Owen ${ }^{8}$, P. Louarn ${ }^{9}$, S. Livi ${ }^{10}$, T. Horbury ${ }^{5}$, N. André ${ }^{9}$, V. Angelini ${ }^{5}$, V. Evans ${ }^{5}$, A. Fedorov ${ }^{9}$, V. Genot ${ }^{9}$, B. Lavraud ${ }^{11,9}$, L. Matteini ${ }^{5}$, D. Müller ${ }^{12}$, H. O’Brien ${ }^{5}$, O. Pezzi ${ }^{13,14,15}$, A. P. Rouillard ${ }^{9}$, L. Sorriso-Valvo ${ }^{15,16}$, A. Tenerani ${ }^{17}$, D. Verscharen ${ }^{8,18}$, and I. Zouganelis ${ }^{19}$

${ }^{1}$ National Institute for Astrophysics (INAF) - Institute for Space Astrophysics and Planetology (IAPS), Via Fosso del Cavaliere, 100, 00133 Rome, Italy e-mail: raffaella.damicis@inaf.it

2 Advanced Heliophysics, Pasadena, CA, USA

3 National Institute for Astrophysics (INAF) - National Observatory of Turin (OATo), Via Osservatorio, Pino Torinese, Turin, Italy

${ }^{4}$ Italian Space Agency (ASI), via del Politecnico snc, 00133 Rome, Italy

5 Department of Physics, Imperial College London, London SW7 2AZ, UK

${ }^{6}$ UCLA Earth Planetary and Space Sciences Department, Los Angeles, CA, USA

7 Istituto Nazionale di Geofisica e Vulcanologia (INGV), Via di Vigna Murata 605, 00143 Rome, Italy

Mullard Space Science Laboratory, Holmbury St Mary RH5 6NT, UK

9 Institut de Recherche en Astrophysique et Planétologie, Université Toulouse III—Paul Sabatier, CNRS, CNES, Toulouse, France

Southwest Research Institute, San Antonio, TX

11 Laboratoire d'Astrophysique de Bordeaux, Univ. Bordeaux, CNRS, Pessac, France

12 European Space Agency, ESTEC (SCI-S), PO Box 299, Noordwijk 2200 AG, The Netherlands

13 Gran Sasso Science Institute (GSSI), Viale F. Crispi 7, 67100 L'Aquila, Italy

14 INFN/Laboratori Nazionali del Gran Sasso, Via G. Acitelli 22, 67100 L'Aquila, Italy

15 Istituto per la Scienza e Tecnologia dei Plasmi, Consiglio Nazionale delle Ricerche, Via Amendola 112/D, 70126 Bari, Italy

16 Swedish Institute of Space Physics, Ångström Laboratory, Lägerhyddsvägen 1, SE-751 21 Uppsala, Sweden

17 The University of Texas at Austin, 2515 Speedway Austin, TX 78712

18 Space Science Center, University of New Hampshire, 8 College Road, Durham NH 03824, USA

19 European Space Agency (ESA), European Space Astronomy Centre (ESAC), Camino Bajo del Castillo s/n, 28692 Villanueva de la Cañada, Madrid, Spain

Received , ; accepted ,

\section{ABSTRACT}

Context. Turbulence dominated by large amplitude nonlinear Alfvén-like fluctuations mainly propagating away from the Sun is ubiquitous in high speed solar wind streams. Recent studies have shown that also slow wind streams may show strong Alfvénic signatures, especially in the inner heliosphere.

Aims. The present study focuses on the characterisation of an Alfvénic slow solar wind interval observed by Solar Orbiter on July $14-18,2020$ at a heliocentric distance of 0.64 AU.

Methods. Our analysis is based on plasma moments and magnetic field measurements from the Solar Wind Analyser (SWA) and Magnetometer (MAG) instruments, respectively. We compare the behaviour of different parameters to characterise the stream in terms of the Alfvénic content and magnetic properties. We perform also a spectral analysis to highlight spectral features and waves signature using power spectral density and magnetic helicity spectrograms, respectively. Moreover, we reconstruct the Solar Orbiter magnetic connectivity to the solar sources via both a ballistic and a Potential Field Source Surface (PFSS) model.

Results. The Alfvénic slow wind stream described in this paper resembles in many respects a fast wind stream. Indeed, at large scales, the time series of the speed profile shows a compression region, a main portion of the stream and a rarefaction region, characterised by different features. Moreover, before the rarefaction region, we pinpoint several structures at different scales recalling the spaghetti-like flux-tube texture of the interplanetary magnetic field. Finally, we identify the connections between Solar Orbiter in situ measurements, tracing them down to coronal streamer and pseudostreamer configurations.

Conclusions. The characterisation of the Alfvénic slow wind stream observed by Solar Orbiter and the identification of its solar source are extremely important aspects to understand possible future observations of the same solar wind regime, especially as solar activity is increasing toward a maximum, where a higher incidence of this solar wind regime is expected.

Key words. interplanetary medium - solar wind - methods: data analysis - magnetohydrodynamics (MHD) - turbulence

\section{Introduction}

The first observation of a peculiar slow solar wind (Marsch et al. 1981) occurred during the ascending phase of solar cycle 21 around 0.29 AU (Helios perihelion). This stream was considered an isolated case until D'Amicis et al. (2011) proved that it is a very common solar wind regime, with a high occurrence rate at 
1 AU especially during the maximum of solar cycle (D'Amicis and Bruno 2015; D'Amicis et al. 2021b). The striking features of this kind of slow wind were the pronounced differential speeds between proton and alpha particles bulk flows and the large proton temperature anisotropies (Marsch et al. 1981). These results were accompanied by the typical signature of Alfvénic fluctuations, namely a strong correlation between velocity and magnetic field vectors, with the sign corresponding to that of Alfvén waves propagating away from the Sun (outward modes), nearly constant magnetic field magnitude and low plasma compressibility (e.g. Belcher et al. 1969; Belcher \& Davis 1971; Belcher \& Solodyna 1975). The above features are similar to what is observed in fast wind streams but different from what had been seen in earlier observations of slow wind at solar minimum. The similarities with the fast wind were also proved statistically on a wide range of parameters (D'Amicis and Bruno 2015; D'Amicis et al. 2019). As a consequence, the standard classification of the solar wind according to the flow speed should be used with caution and should be accompanied by other indicators like, for instance, the Alfvénic content of the fluctuations (D'Amicis et al. 2021a, and references therein).

Alfvénic outward modes coexist with fluctuations propagating towards the Sun (inward modes), although their origin depends on their location with respect to the Alfvénic radius, the latter being the critical distance where the solar wind becomes super-Alfvénic, ranging between 10 to 30 solar radii $\left(R_{\odot}\right)$ (Goelzer et al. 2014). The nonlinear interaction between inward and outward modes, present in different amounts in the solar wind (Tu et al. 1984), produces a turbulent cascade which is well-described by a typical power-law spectrum (as first observed by Coleman 1968), with a slope in the inertial range between $-5 / 3$ (Kolmogorov 1941) and $-3 / 2$ (Iroshnikov 1963; Kraichnan 1965). Alfvénic intervals typically show higher power with respect to non-Alfvénic streams, due to the stronger fluctuations in the velocity and magnetic fields (D'Amicis et al. 2019, 2020). Moreover, for these intervals, the large scales of the turbulent cascade are characterised by a $1 / f$ power law, as expected for fluctuations that are scale-independent, which is separated from the inertial range by a break around typical scales between minutes and hours. The specific location of the break between the $1 / f$ range and the inertial range depends both on heliocentric distance and on the turbulent age (D'Amicis et al. 2019). Despite several mechanisms have been proposed, the nature of the $1 / f$ spectrum is still not fully understood. For example, it has been attributed to the superposition of uncorrelated samples of turbulence of different solar origin (Matthaeus $\&$ Goldstein 1986), to the presence of an inverse cascade of low-frequency modes (Dmitruk \& Matthaeus 2007), or to the contribution of outward propagating modes reflected by largescale solar wind gradients in the extended solar corona (Verdini et al. 2012). The presence of the $1 / f$ scaling is also associated with the saturation of the magnetic field fluctuations to the amplitude of the local magnetic field (Matteini et al. 2018; Bruno et al. 2019; D'Amicis et al. 2020; Perrone et al. 2020). Conversely, non-Alfvénic streams show a Kolmogorov-like scaling from large to inertial scales, even if a $1 / f$ power law can be found for long enough intervals which can properly capture the low-frequency spectral properties (Bruno et al. 2019). Finally, a steeper power law can be observed in the turbulent cascade beyond ion scales, often called kinetic or dissipation range. The latter is separated from the inertial range by another break in the magnetic field spectrum. It is not clear whether its location depends on the Alfvénic content of the fluctuations.
The nature of the fluctuations that populate the ion scales near the kinetic break was studied by several authors (see e.g. Goldstein et al. 1994; Leamon et al. 1998; Hamilton et al. 2008), who provided the first inferences of the presence of Kinetic Alfvén Waves (KAWs) in the solar wind kinetic range. However, a Fourier analysis as used by these authors is unable to separate different types of small-scale waves. For instance, this method only samples left-hand polarised Alfvén/ion-cyclotron waves (ICWs) during time intervals in which the background magnetic field is quasi-parallel to the radial direction. ICWs are generally intermixed with KAWs, which have a broader range of propagation directions and are thus more frequently sampled. Since the classical Fourier analysis provides information only in the frequency domain, providing thus global information along the whole spatial range spanned by the spacecraft, it cannot single out spatial structures or wave packets with different characteristics possibly crossed by the spacecraft. This limitation was overcome more recently by a wavelet transform-based analysis technique (first suggested by Horbury et al. 2008), which has been extensively used (He et al. 2011, 2012a,b; Podesta \& Gary 2011; Telloni et al. 2012, 2019, 2020) to study the normalised magnetic helicity (Matthaeus \& Goldstein 1982). Indeed, this technique looks at the polarisation state of the fluctuations on a plane perpendicular to the sampling direction and for different pitch angles with respect to the local mean magnetic field orientation. Both L1 observatories and Ulysses measurements confirmed simultaneous signatures of right-handed polarised KAWs (or whistler waves) at large angles of propagation with respect to the local mean magnetic field, $B_{0}$, and left-handed ICWs outward propagating almost (anti-)parallel to $B_{0}$. Moreover, Telloni et al. $(2019,2020)$ have shown that the amplitude of the Alfvénic fluctuations at fluid scales, rather than other quantities (such as e.g. the solar wind speed), is the key parameter in driving the generation of the ICWs at kinetic scales. They also suggested that these waves are generated through proton cyclotron instability, triggered by large temperature anisotropies. Finally, ICWs can be identified as the most evident signature of the resonant dissipation of Alfvén waves at frequencies near the gyrofrequency, also in Alfvénic slow solar wind (Telloni et al. 2020).

The Alfvénic content of the solar wind is a characteristic of the plasma which strongly depends on heliocentric distance, decreasing with increasing distance. Parametric instability has been invoked as a possible mechanism responsible for the gradual decrease of the Alfvénic correlation causing an increasing importance of inward-propagating Alfvénic fluctuations with respect to the main outward-propagating component (e.g. Malara et al. 2000; Del Zanna et al. 2001; Matteini et al. 2010; Tenerani \& Velli 2013; Primavera et al. 2019). In particular, the Alfvénic signature of the fluctuations observed close to the Sun (at 0.3 AU for Helios and at closer distances with Parker Solar Probe) in the slow wind appears to be generally lost when approaching Earth. However, observations by Wind at $1 \mathrm{AU}$, especially during the maximum of solar cycle 23 , are rather at odds with previous observations since large Alfvénic fluctuations are observed also in the slow wind, as highlighted in D'Amicis et al. (2011); D'Amicis and Bruno (2015); D'Amicis et al. (2019). Indeed, these streams strongly resemble, except for their velocity, the fast wind. This suggests a similar origin for Alfvénic streams: open field regions on the solar surface, namely coronal holes (Stansby et al. 2020; Perrone et al. 2020; D'Amicis et al. 2020). Although the source regions of the Alfvénic slow wind are still an open question, regions of anomalous, larger than average, expansion rate of magnetic flux tubes near the Sun appear to be a leading candidate (D'Amicis et al. 2021a). As shown in 
Panasenco \& Velli (2013); Panasenco et al. (2019, 2020) such regions may form easily when large scale pseudostreamers are present in the corona. Pseudostreamers (PSs) are characterised by multipolar regions of confined field, that open into interplanetary space in a unipolar fashion. Pseudostreamers therefore separate coronal holes with the same polarity, contrary to helmet streamers (HSs) that form between coronal holes with opposite polarities. The main differences between PSs and HSs are 1) the height of a pseudostreamer cusp or X-point is found to be lower in the corona, by at least factor of 2, with respect to the height of the Y-type neutral point of the HS tip (Wang et al. 2012; Panasenco \& Velli 2013); 2) the presence of a welldeveloped current sheet above the HS tip and outward, but the absence of an associated polarity reversal or current sheet in the outer corona pseudostreamer; 3 ) the presence of at least two (and possibly four, but always even-numbered) neutral lines at the PS base, meaning that twin filaments may be harbored in PS lobes. Field lines opening into space from the neighborhood of pseudostreamer lobes tend to have non-monotonic, large expansions, as illustrated by coronal magnetic funnels in Panasenco et al. (2019), and the presence of filament channels in the pseudostreamer lobes increases the probability and strength of the non-monotonic expansion and divergence of the open magnetic field from pseudostreamer configurations in three-dimensional modeling.

The connections between the physical processes occurring at the Sun and the features observed locally in the solar wind can now be studied thanks to the Solar Orbiter mission (Müller et al. 2020). Launched in February 2020, Solar Orbiter is a unique mission to both study in detail the physics of the solar wind in situ, thanks to four high-time-resolution instruments for plasma, fields and energetic particles (Walsh et al. 2020), and its source regions, through high-resolution remote-sensing observations by six different instruments (Auchère et al. 2020). Indeed, the combination of both in situ measurements and remote sensing observations (García Marirrodriga et al. 2021), for the first time in a single spacecraft in the inner heliosphere, will allow unprecedented magnetic connectivity analysis between the solar atmosphere and the inner heliosphere (Zouganelis et al. 2020).

The Alfvénic slow solar wind is a statistically important solar wind regime, both in the inner heliosphere and at the Earth and in different phases of the solar cycle (D'Amicis et al. 2021a,b). Although observed in previous missions, it is at present in the limelight due to the very recent observations by Parker Solar Probe, which observed several streams of Alfvénic slow wind at distance close to the Sun never reached before. The focus of this paper is the characterisation of an Alfvénic slow wind interval observed very recently by Solar Orbiter at about 0.64 AU. Data selection will be presented in Section 2. Section 3 will be devoted to a global description of the stream in situ characteristics, while the connection between the in situ measurements and the solar source will be discussed in Section 4. A summary discussion of the results will be presented in Section 5 .

\section{Data selection}

The study presented in this paper was performed over a time interval ranging between 14 July 00:00 UT (universal time) to 18 July 12:00 UT, 2020, during which Solar Orbiter crossed an Alfvénic slow wind stream. A crucial set of measurements for our study is provided by the Solar Wind Analyser (SWA) suite of instruments (Owen et al. 2020), consisting of an Electron Analyser System (SWA-EAS), a Proton and Alpha Particle Sensor (SWA-PAS), and a Heavy Ion Sensor (SWA-HIS) which

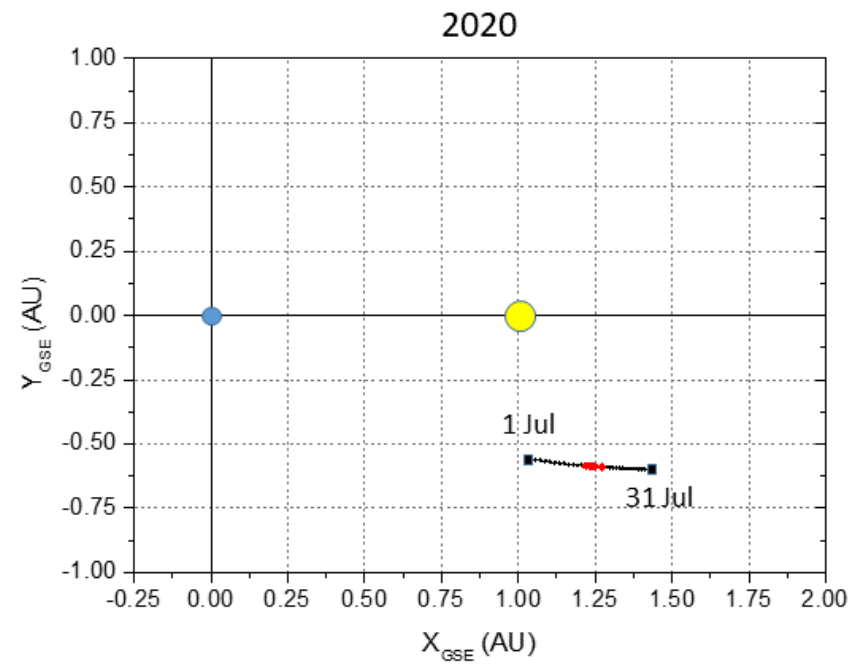

Fig. 1. Position of Solar Orbiter during July 2020 (black dots). The plot shows the projection of the orbit on the ecliptic plane in GSE (geocentric solar ecliptic) coordinates, so that Earth is at $[0,0]$ (blue dot), the Sun is at [1,0] (yellow dot). Red dots highlight the position of the s/c during the selected interval.

are jointly served by a data processing unit (SWA-DPU). This analysis, in particular, is based on solar wind measurements derived from SWA-PAS which is an electrostatic analyser with a confined field of view $\left(-24^{\circ}\right.$ to $+42^{\circ} \times \pm 22.5^{\circ}$ around the expected solar wind arrival direction). SWA-PAS measures the full $3 \mathrm{D}$ velocity distribution function (VDF) of the protons and alpha particles arriving at the instrument in the energy range from $200 \mathrm{eV} / \mathrm{q}$ to $20 \mathrm{keV} / \mathrm{e}$. From the VDF, ground moments (e.g. number density, velocity vector and temperature computed from the pressure tensor) are derived and provided at $4 \mathrm{sec}$ resolution. The SWA-PAS database was downloaded from the AMDA webserver ${ }^{1}$. We also included magnetic field measurements from the Magnetometer (MAG) instrument (Horbury et al. 2020a), downloaded from the ESA Solar Orbiter archive ${ }^{2}$ and averaged at the plasma sampling time.

Fig. 1 shows the projection of Solar Orbiter orbit, during July 2020 (black dots), on the ecliptic plane. The plot is given in GSE (geocentric solar ecliptic) coordinates, so that Earth is at $[0,0]$ (blue dot) and the Sun is at $[1,0]$ (yellow dot). The position of the $\mathrm{s} / \mathrm{c}$ in the period of our analysis is highlighted in red. During that period, after its first perihelion on June 15 , the s/c was progressively moving away from the Sun.

\section{Global in situ description of the stream}

Although the Alfvénic slow wind has mainly been observed and studied during the maximum of the solar cycle, evidences of the presence of this solar wind regime occur also at the minimum of the solar cycles (D'Amicis et al. 2021b). Indeed, Solar Orbiter has been embedded in a stream of Alfvénic slow wind in July 2020 , during the minimum of solar cycle 24 . In the following, we will give a complete description of this interval in terms of Alfvénic content, plasma features and spectral properties. 


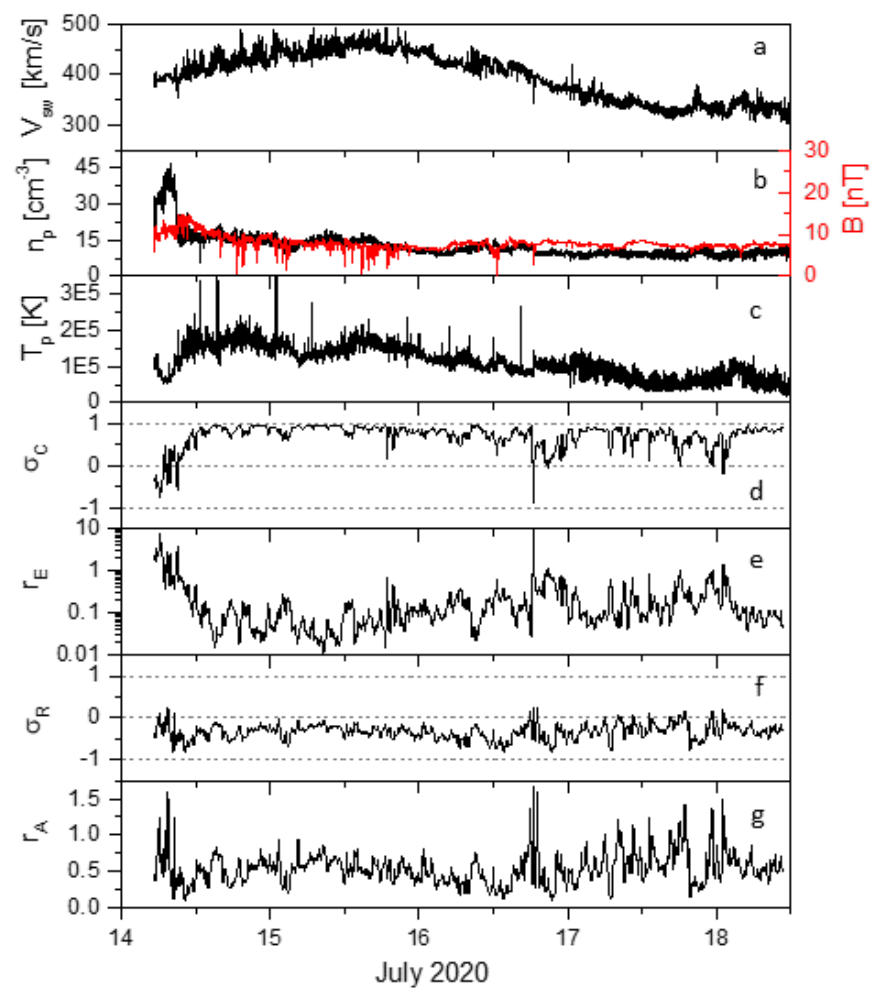

Fig. 2. Time series of plasma and other relevant parameters characterising Alfvénicity in the slow wind stream observed by Solar Orbiter at a heliocentric distance of $0.64 \mathrm{AU}$ : solar wind speed, $V_{s w}[\mathrm{~km} / \mathrm{s}]$ (a); proton number density, $n_{p}\left[\mathrm{~cm}^{-3}\right]$ (black) and magnetic field magnitude, $B$ (red) (b); proton temperature, $T_{p}[\mathrm{~K}]$ (c); the normalised cross-helicity, $\sigma_{C}(\mathrm{~d})$; the Elsässer ratio, $r_{E}(\mathrm{e})$; the normalised residual energy, $\sigma_{R}(\mathrm{f})$; and the Alfvén ratio, $r_{A}(\mathrm{~g})$. The derived quantities are computed at 30 min scale.

\subsection{Alfvénic slow wind}

Fig. 2 shows an overview of the Alfvénic slow wind stream observed by Solar Orbiter at a heliocentric distance of 0.64 AU. The same time interval has been also studied in another paper of this special issue by Louarn et al. (2021) focusing on different aspects and in particular on the characterisation of the proton distribution functions observed by PAS. During the selected interval, the speed values (panel a) are less than $500 \mathrm{~km} / \mathrm{s}$ thus identifying a slow stream. However, the speed profile is similar to that of a fast wind stream. Indeed, we observe a compression region at the leading edge of the stream and a rarefaction at the trailing edge. The compression region (14.25 - 14.5 July) is characterised by an increase in the proton number density and magnetic field magnitude (see panel $b$ ). The main portion of the stream, extending from approximately 14.5 to 16 July, displays higher speed and large amplitude fluctuations and approximately constant $n_{p}$ and $B$. Then, a rarefaction region appears, which is characterised by a gradual decrease of the flow speed and smaller amplitude fluctuations. Moreover, the proton temperature profile, $T_{p}$ (panel c), follows the V-T relationship (see e.g. Burlaga \& Ogilvie 1970; Lopez \& Freeman 1986; Matthaeus et al. 2006; Elliott et al. 2012; Perrone et al. 2019). This is indeed larger in the main portion of the stream than in the rarefaction region. The characterisation of the different portions of the stream will be discussed in more details in the subsection 3.2.

\footnotetext{
1 http://amda.irap.omp.eu/

2 http://soar.esac.esa.int/soar
}

The speed profile exhibits velocity variations of the order of $\pm 35-50 \mathrm{~km} / \mathrm{s}$, on typical timescales of $6-10 \mathrm{~h}$, that can be attributed to the so called 'microstreams' as first observed by Ulysses in the fast polar wind (Neugebauer 1995, 1997) and in Helios data close to the Sun (Horbury et al. 2018). Neugebauer (2012) interpreted these structures as the in situ signature of reconnection jets, due to newly emerging bright-point loops with previously open magnetic fields (see e.g. Subramanian et al. 2010) present in the chromospheric network. Similar largescale structures were observed also in the properties of the proton plasma quantities and in the Alfvénicity (Borovsky 2016).

Following D'Amicis et al. (2021a) and references therein, we first study the Alfvénic content of the fluctuations. Alfvénicity can be studied using the Elsässer variables (Elsässer 1950), introduced for the first time in the interplanetary data analysis by Tu et al. (1989) and Grappin et al. (1991). The Elsässer variables are defined as follows: $z^{ \pm}=v \pm b$ where $b$ is the magnetic field expressed in Alfvén units $\left(b=B /(4 \pi \rho)^{1 / 2}\right.$, with $\rho$ being the mass density and $B$ is the magnetic field). The sign in front of $b$ is given by $\operatorname{sign}\left(-k \cdot B_{0}\right)$, where $k$ is the wave vector and $B_{0}$ is the ambient magnetic field:

i) for a field directed outward with respect to the Sun, a negative (positive) correlation indicates a mode propagating away from (toward) the Sun. In this case, Elsässer variables are defined as $z^{+}=v-b$ and $z^{-}=v+b$ for outward and inward modes, respectively;

ii) when the field is directed towards the Sun, the correlation sign reverses with respect to the previous cases. However, the scientific community has agreed to define $z^{+}\left(z^{-}\right)$always as outward (inward)-directed Alfvénic fluctuations. To do this, the magnetic field is rotated by $180^{\circ}$, every time that it is directed towards the Sun (Roberts et al. 1987; Bruno \& Bavassano 1991; Grappin et al. 1991). Then, in this case $b \rightarrow-b$ and Elsässer variables are defined as $z^{+}=v+b$ and $z^{-}=v-b$.

In this stream the magnetic field is essentially outward-directed as we will see in the next section.

In Fig. 2, as first introduced by Tu \& Marsch (1995), we analyze the energy associated with $z^{+}$and $z^{-}$modes, namely $e^{+}$ (red) and $e^{-}$(black) at $30 \mathrm{~min}$ scale as solar wind fluctuations show a strong Alfvénic character at this scale (Tu \& Marsch 1995; Bavassano et al. 1998). In particular, we focus on their (normalised) difference, namely the normalised cross-helicity, $\sigma_{c}=\left(e^{+}-e^{-}\right) /\left(e^{+}+e^{-}\right)$(panel d), and their ratio, the Elsässer ratio, $r_{E}=e^{-} / e^{+}$(panel e). As expected, there is a clear predominance of $e^{+}$respect to $e^{-}\left(\sigma_{c}\right.$ close to 1 and $\left.r_{E} \ll 1\right)$ indicating a dominance of outward propagating Alfvén modes. Moreover, $\sigma_{c}$ profile evolves along the stream with higher values in the main portion of the stream than in the rarefaction region. At the same time, in the two regions, $r_{E}$ changes considerably with $e^{+} \gg e^{-}$ in the main portion. On the other hand, we also evaluate the normalised residual energy: $\sigma_{R}=\left(e^{v}-e^{b}\right) /\left(e^{v}+e^{b}\right)$ (panel f), and the Alfvén ratio, $r_{A}=e^{v} / e^{b}$ (panel g), where $e^{v}$ and $e^{b}$ are the kinetic and magnetic energies, respectively. These quantities indicate the imbalance between kinetic and magnetic energy of the fluctuations. Fig. 2 shows overall an imbalance in favor of magnetic energy $\left(\sigma_{R}\right.$ is negative implying $e^{b}>e^{v}$ and $\left.r_{A}<1\right)$. Although $r_{A}$ is very close to unity near the Sun $(0.3 \mathrm{AU})$, it appreciably decreases with increasing radial distance in near-ecliptic solar wind (e.g. Bruno et al. 1985; Marsch \& Tu 1990), reaching an asymptotic value of 0.5 around $1 \mathrm{AU}$. The departure from energy equipartition (namely $e^{b} \simeq e^{v}$ as expected for an ideal Alfvén wave) might be due to the turbulence evolution (see e.g. Grappin et al. 1991; Roberts et al. 1992), to the effect of solar 


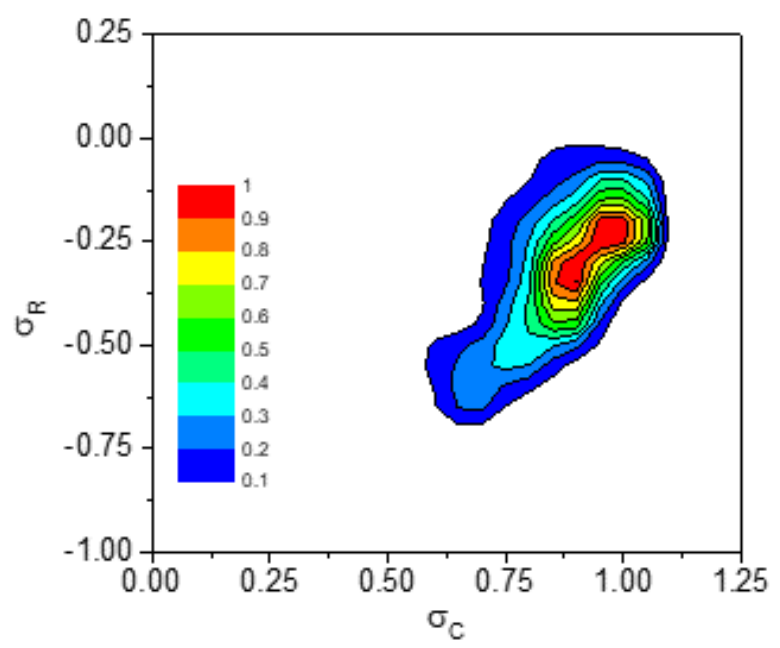

Fig. 3. 2D contour plot of $\sigma_{C}$ vs $\sigma_{R}$ for the Alfvénic slow wind stream observed by Solar Orbiter at $0.64 \mathrm{AU}$. The color bar represents the percentage with respect to the maximum value. Values of $\sigma_{C}>1$ are artifacts of the 2D contour plot interpolation.

wind structures (Tu \& Marsch 1993) or also to effects related to pressure anisotropy and ion differential streaming (Bavassano \& Bruno 2000).

The degree of the $v-b$ correlation depends not only on the type of wind, but also on the radial distance from the Sun and on the time scale of the fluctuations (Bruno \& Carbone 2013). Using 2D histograms of $\sigma_{C}-\sigma_{R}$ (Bavassano et al. 1998), Bruno et al. (2007) were able to characterise the turbulence state of solar wind fluctuations of Helios observations and showed that at short heliocentric distances $(\sim 0.3 \mathrm{AU})$ the turbulent population is largely dominated by Alfvénic fluctuations, characterised by high values $\sigma_{C}$ and equipartition of energy. However, as the wind expands, Alfvénic fluctuations are depleted and another population, which displays lower values of $\sigma_{C}$ and a clear imbalance in favor of magnetic energy, becomes visible and easily distinguishable from the Alfvénic population (Wicks et al. 2013). In the top panel of Fig. 3, we characterise the state of turbulence of this stream of Alfvénic slow wind in a similar way. A predominance of outward modes is observed, characterised by a magnetic energy excess since the distribution extends over the quadrant $\sigma_{C}>0-\sigma_{R}<0$, in agreement with existing literature (Bruno et al. 1985; Bavassano et al. 1998, 2000; D’Amicis et al. 2007, 2011; D'Amicis and Bruno 2015). Moreover, the main feature is a pronounced peak corresponding to Alfvénic fluctuations $\left(\sigma_{c}\right.$ close to 1 and $\sigma_{R}$ close to 0 ) and a tail towards lower values of $\sigma_{C}$, and more imbalanced magnetic structures (lower negative values of $\sigma_{R}$ ) (Bavassano et al. 1998), features very similar to the ones of the fast wind observed by Helios at $0.65 \mathrm{AU}$ (Bruno et al. 2007).

\subsection{Identifying different portions of the stream}

As anticipated in the previous subsection, different regions, with well-defined features, can be identified in this Alfvénic slow stream, as shown in Fig. 4. The solar wind speed is inserted in panel (a) for completeness. Panel (b) shows the $v-b$ correlation coefficient, $\rho_{v b}$, computed at $30 \mathrm{~min}$ scale using a running window, as another parameter to measure Alfvénicity. It is defined as the ratio between the covariance of the two variables $v$ and $b$, divided by the product of their standard deviations:

$\rho_{v b}=\frac{\sum_{j}\left(V_{j}-\bar{V}\right)\left(B_{j}-\bar{B}\right)}{\sqrt{\sum_{j}\left(V_{j}-\bar{V}\right)^{2}\left(B_{j}-\bar{B}\right)^{2}}}$

where $V_{j}$ and $B_{j}$ are the velocity and magnetic field single measurements and $\bar{V}$ and $\bar{B}$ are the velocity and magnetic field averages in each $30 \mathrm{~min}$ window. In principle, this formula should be applied to every component of the velocity $\left(V_{i}\right)$ and magnetic fields $\left(B_{i}\right)$ (with $i=R, T, N^{3}$ ) and then we derive the total correlation coefficient as the average from the three components, $\sum_{i} \rho_{v b_{i}} / 3$. However, for the sake of simplicity, we consider only the $\mathrm{N}$ component since more Alfvénic than the other two (Tu et al. 1989). As in previous studies, $\rho_{v b}$ indicates that the main portion of the stream (red box of Fig. 4) is the most Alfvénic part (very high absolute values of $\rho_{v b}$ ), while Alfvénicity decreases when moving towards the rarefaction region (blue box).

The amplitude of velocity and magnetic fluctuations can be measured by their respective variances (panel c), defined as $\sigma_{V}^{2}=\sigma_{V_{R}}^{2}+\sigma_{V_{T}}^{2}+\sigma_{V_{N}}^{2}$ (black) and $\sigma_{B}^{2}=\sigma_{B_{R}}^{2}+\sigma_{B_{T}}^{2}+\sigma_{B_{N}}^{2}$ (red), normalised to the square of their average fields. Both quantities are larger in the main portion of the stream compared to the rarefaction region (blue box in Fig. 4), as also shown by Carnevale et al. (2021). The higher variance of the fluctuations in the main portion indicates the presence of large amplitude Alfvénic fluctuations. The transition between the main portion and the rarefaction region is such that the Alfvénic content of the fluctuations along with the amplitude of fluctuations decrease considerably (Ko et al. 2018).

To understand the spatio-temporal evolution of the magnetic field vector, we study the changes experienced by the vector orientation. Panel (d) displays the vector displacement, $|\delta \mathbf{B}|$, between each magnetic field instantaneous direction and an arbitrary fixed direction, normalised to the average magnetic field, $\langle B\rangle$, defined as:

$|\delta \mathbf{B}(t)| /\langle B\rangle=\sqrt{\sum_{i}\left(B_{i}(t)-B_{i}\left(t_{0}\right)\right)^{2}} /\langle B\rangle$

with $i=R, T, N$, as first studied by Bruno et al. (2004). The arbitrary fixed direction was chosen to be the direction of the first vector of the time series, $B\left(t_{0}\right)$. However, the result of this kind of analysis does not depend on this assumption. The vector displacement provides important information on solar wind fluctuations, as shown in Bruno et al. (2004). Indeed, by looking how the magnetic field orientation fluctuates in space, these authors found that the magnetic fluctuations are characterised by the contribution of two components: small-amplitude and highfrequency fluctuations superimposed on a larger-amplitude lowfrequency background structure. Thus, this quantity is sensitive to both propagating Alfvénic fluctuations and advected structures (e.g., flux tubes), the two main ingredients of solar wind turbulence (Bruno \& Carbone 2013). Panel d clearly shows different regimes within the stream. Indeed, the main portion is dominated by directional fluctuations which are generally large, i.e. the Alfvénic component. The other portions of the stream, less (but still) Alfvénic, shows smaller fluctuations and fewer large and quick directional jumps. This is in agreement with the results by Bruno et al. (2004) and their interpretation according

\footnotetext{
${ }^{3} V_{i}$ and $B_{i}$ are in the heliographic Radial Tangential Normal (RTN) coordinate system, where $\mathrm{R}$ points away from the Sun toward the spacecraft, $T$ is the cross product of the Sun's spin axis and $R$, and $N$ completes the right-handed triad.
} 


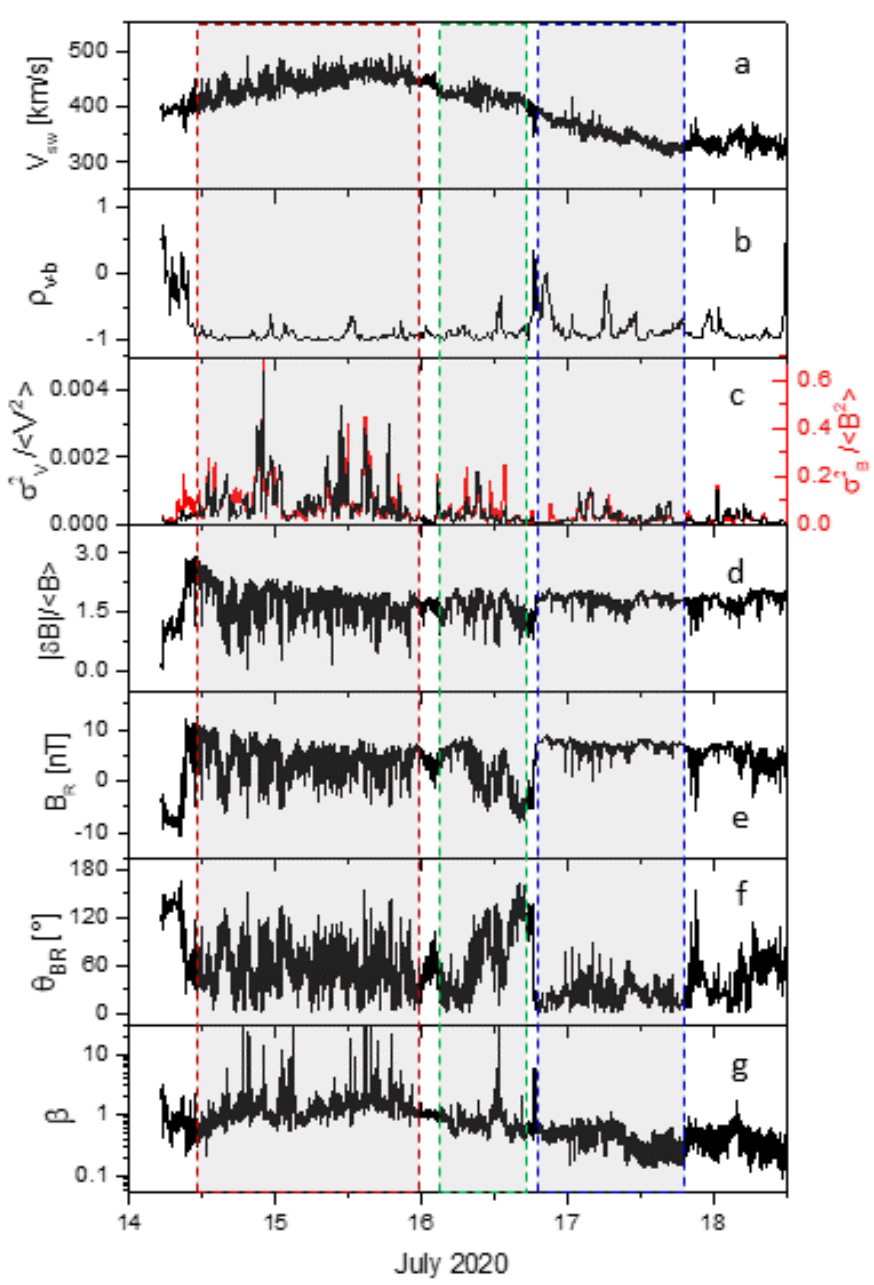

Fig. 4. Time series of relevant parameters: solar wind speed, $V_{s w}[\mathrm{~km} / \mathrm{s}]$ (a); $v-b$ correlation coefficient, $\rho_{v b}$ (b), computed at 30 min scale (b); $V$ and $B$ variances normalised to the square of their respective mean fields, $\sigma_{V}^{2} /\left\langle V^{2}\right\rangle$ (black) and $\sigma_{B}^{2} /\left\langle B^{2}\right\rangle$ (red) (c); magnetic field vector displacement, $|\delta B| /\langle B\rangle$ (d); radial component, $B_{R}$ [nT], in RTN coordinate system (e); angle between the magnetic field direction and the radial direction, $\theta_{B R}(\mathrm{f})$; and plasma beta, $\beta(\mathrm{g})$. The red and blue boxes identify the main portion of the stream and the rarefaction region, respectively. The green box identifies a region characterised by the presences of structures, that will be studied in detail in Section 3.4.

to which the large jumps correspond to tangential discontinuities marking the borders between adjacent flux tubes. In each flux tube the presence of Alfvénic fluctuations makes the magnetic field vector randomly wander about a local field direction. The effect of the directional jumps is to move the tip of the fluctuating vector from one particular average direction to another one (see also section 3.4 and Figure 10).

The passage from one region to the other one is also marked by the different behaviour of the radial component, $B_{R}$, of the magnetic field (panel e), characterised by the presence of large and intermittent polarity reversals, called switchbacks, especially in the main portion of the stream. In the rarefaction region, the field is almost radial in agreement with previous studies (e.g. Orlove et al. 2013) and the switchback activity is much weaker with much fewer inversions, and smaller amplitudes than the ones observed in the main portion of the stream. These polarity reversals, observed very recently by Parker Solar Probe, are Sshaped magnetic structures (Dudok De Wit et al. 2020; Chhiber et al 2020; Mozer et al. 2020; McManus et al. 2020), naturally associated with localised radial velocity enhancements (Kasper et al. 2019; Horbury et al. 2020b) having oscillation amplitudes comparable to the magnitude of the magnetic field (Bale et al. 2019). Although previously observed in the fast wind, by missions at different heliocentric distances (Behannon et al. 1981; Tsurutani et al. 1994; Kahler et al. 1996; Balogh et al. 1999; Yamauchi et al. 2002; Landi et al. 2006; Matteini et al. 2014; Borovsky 2016), the switchbacks are ubiquitous features of the Alfvénic slow wind in the inner heliosphere (e.g. D'Amicis et al. 2021a, and references therein).

The behaviour of the angle between the magnetic field and the radial direction, $\theta_{B R}$ (panel f), provides similar information. It shows large fluctuations around about $60^{\circ}$ within the main portion of the stream, while it is very small (almost radial field) in the rarefaction region. This is a clear indication that, in the main portion of the stream, the magnetic field direction is changing due to the presence of large amplitude Alfvénic fluctuations.

Panel ( $\mathrm{g}$ ) shows the behaviour of the plasma beta, $\beta$, computed as the ratio between the thermal pressure, $p_{k}$ and the magnetic pressure, $p_{m}$ or analogously as $V_{t h}^{2} / V_{A}^{2}$ where, $V_{t h}$, the thermal speed, is defined as $\left(2 k_{B} T_{p} / m_{p}\right)^{1 / 2}$, and the $V_{A}$, the Alfvén speed, as $B /(4 \pi \rho)^{1 / 2}$, with $k_{B}$ the Bolzmann's constant, $T_{p}$ the average proton temperature, and $m_{p}$ the proton mass. The evolution of $\beta$ also identifies the different portion of plasma and, in particular, it shows a transition from values higher than 1 in the main portion of the stream to values well below 1 in the rarefaction region.

It must be noted that the behaviour of the parameters characterising the sub-interval (green box in Fig. 4) between the main portion of the stream and the rarefaction region is rather different from the other two. First of all, this interval is quite Alfvénic but with smaller amplitude of the fluctuations with respect to the main portion of the stream but larger than the rarefaction region as shown in the behaviour of $\sigma_{V}^{2} /\left\langle V^{2}\right\rangle$ and $\sigma_{B}^{2} /\left\langle B^{2}\right\rangle$, and $|\delta \mathbf{B}(t)| /\langle B\rangle$. The radial component of the magnetic field does not show switchbacks, rather a strong $B_{R}$ polarity inversion at odds with the dominant (positive) polarity of the stream. This reflects in a sudden change of $\theta_{B R}$ between very small values (almost aligned field) and $\theta_{B R} \sim 120^{\circ}$. A detailed analysis of this region will be performed in section 3.4. The difference in the switchback activity before 16 July 00:00 UT and after 16 July 18:00 UT, related to dramatic changes in the solar source region magnetic topology, will be discussed in Section 4.

To conclude this subsection, we analyzed the sharp discontinuity at day 16.766 (see Figure 2 and 4) which marks the beginning of the rarefaction region and has been identified as a reconnection exhaust crossing. See also Lavraud et al. (2021), this special issue. This can be clearly seen in Figure 5, where plasma and magnetic field observations are shown for the time interval around the exhaust. Observations are reported in the $L M N$ coordinates, with $\mathbf{L}(-0.81 ;-0.25 ;-0.52)$ being the direction of the anti-parallel component of the magnetic field $\left(B_{L}\right)$, $\mathbf{M}(0.31 ;-0.95 ;-0.022)$ the direction of $\mathrm{X}$ line (and of the guide field $\left.B_{M}\right)$ and $\mathbf{N}(-0.49 ;-0.18 ; 0.85)$ being along the normal to the current sheet. The $\mathbf{N}$ normal to the current sheet is computed by means of the Minimum Variance (MV) analysis performed across the exhaust (e.g. Sonnerup \& Cahill 1967), $\mathbf{M}$ is computed as $\mathbf{M}=\mathbf{N} \times\left(\mathbf{B}_{\mathbf{A}}-\mathbf{B}_{\mathbf{B}}\right) /\left|\mathbf{B}_{\mathbf{A}}-\mathbf{B}_{\mathbf{B}}\right|$, where $\mathbf{B}_{\mathbf{A}}$ and $\mathbf{B}_{\mathbf{B}}$ are the magnetic field vectors tangential to the current sheet measured on the two sides of the exhaust, and $\mathbf{L}$ completes the orthogonal triad (e.g. Davis et al. 2006). The plasma and field signatures are characteristic of the crossing of a bifurcated current 
sheet encompassing decelerated plasma flows, which are roughly Alfvénic, with the changes in $\mathrm{V}$ and $\mathrm{B}$ components (panels $\mathrm{b}$ and d) being anticorrelated (correlated) on the leading (trailing) portion of the exhaust. Moreover, such flows are quantitatively consistent with the reconnection model that predicts that two rotational discontinuities are present at the edge of the exhaust. In such case, the plasma flows should vary across the current sheets according to the relation:

$\mathbf{V}_{\text {pred }}=\mathbf{V}_{r e f} \pm\left[\left(1-\alpha_{r e f}\right) /\left(4 \pi \rho_{r e f}\right)\right]^{1 / 2}\left[\left(\rho_{r e f} / \rho\right) \mathbf{B}-\mathbf{B}_{r e f}\right]$

where $\mathbf{B}, \mathbf{v}, \rho$ are the magnetic field vector, plasma flow velocity and mass density, respectively; $P_{\|}$and $P_{\perp}$ are the pressure parallel and perpendicular to the magnetic field and $\alpha=$ $\left(P_{\|}-P_{\perp}\right) 4 \pi / B^{2}$ is the pressure anisotropy factor (Hudson 1970; Paschmann et al. 1970). The subscript 'ref' indicates indicates a dataset point in the ambient plasma in proximity of the discontinuity. In panels $\mathrm{c}$ and $\mathrm{d}$ of 5 , the predicted values obtained by the above relation are reported as black lines, with positive (negative) sign for the trailing (leading) portion of the bifurcated current sheet and using for the leading (trailing) portion the reference point indicated by the left (right) dashed line. The leading and trailing portion predictions merge at about 16.778 . The comparison between the observed and predicted plasma flows variations evidences that the observations are very well in agreement with the quantitative prediction for reconnection. This event would deserve a more detailed analysis that, however, falls outside the scope of the present paper.

\subsection{Spectral analysis}

The identification of the different regions within the Alfvénic slow stream also has implications on the spectral properties, that motivated us to perform a comparative spectral analysis.

\subsubsection{Power spectra}

The first part of the spectral analysis is devoted to a comparative study of power spectra of the regions identified in Fig. 4, using MAG data in normal mode with a sampling time of $0.125 \mathrm{~s}$. Fig. 6 (bottom panel) shows the power spectral density (PSD) of the trace of magnetic field fluctuations of the three regions. The PSD are computed over a one-day interval in the main portion of the stream and in the rarefaction region (15 July 00:00-23.59 UT and from 16 July 19:12 UT to 17 July 19:12 UT, respectively) and over 12 hours in the intermediate region (16 July 07:12-19:12 UT). The time resolution and the length of the interval allow us to clearly identify the three main frequency ranges of the turbulent spectrum, indicated in Fig. 6 as $f^{-\alpha}, f^{-\beta}, f^{-\gamma}$. Indeed, we observe an $1 / f$ scaling at low frequencies. Although its interpretation has been highly debated (e.g. Matthaeus \& Goldstein 1986; Dmitruk \& Matthaeus 2007; Verdini et al. 2012; Tsurutani et al. 2018), it has been recently associated with the saturation of the magnetic field fluctuations to the amplitude of the local magnetic field (Matteini et al. 2018; Bruno et al. 2019; D'Amicis et al. 2020; Perrone et al. 2020). Then, the inertial range is welldescribed by a spectral index between the Kraichnan and the Kolmogorov theoretical scaling. The higher power spectrum of the main portion of the stream is the result of the presence of large amplitude Alfvénic fluctuations characterising this region. Finally, steeper spectra characterise the high frequency part of the spectrum. In particular, the steepest spectrum corresponds to the main portion of the stream characterised by the highest power in the inertial range, highlighting a strong link between

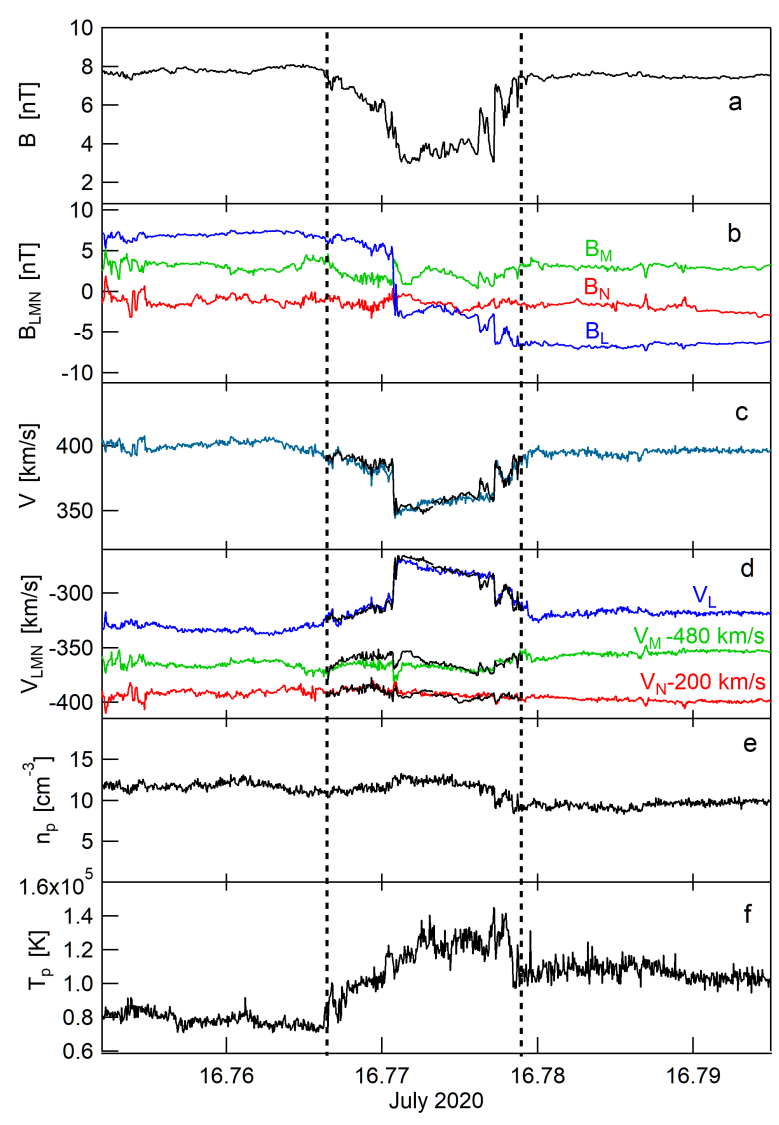

Fig. 5. From top to bottom: the magnetic field magnitude, $B$ (panel a); the magnetic field components in the $L M N$ coordinates system (panel b); the solar wind speed, $V_{s w}$ (panel c); the solar wind velocity components in the $L M N$ coordinates system (panel d); the proton number density, $n_{p}$ (e); and the proton temperature, $T_{p}(\mathrm{~d})$. In panels $\mathrm{c}$ and $\mathrm{d}$, the black lines represent the reconnection model predictions according to Equation 3. The left (right) dashed line indicates the reference point used for the prediction in the leading (trailing) portion of the exhaust.

the inertial and kinetic scales, as first identified by Bruno et al. (2014) and more recently by D'Amicis et al. (2019).

The upper panel of Fig. 6 shows the normalised power spectra of the trace of magnetic field fluctuations, similar to Bruno et al. (2019) and D'Amicis et al. (2020), to better highlight similarities and/or differences between the relative amplitude of the fluctuations and the scaling of the power spectra in the different regimes we identified in this Alfvénic slow stream. The normalised power spectral density is derived in the following way. The amplitude of the fluctuation $\delta B(f)$ at a given frequency $f$ can be computed as:

$\delta B(f)=\sqrt{2 f P_{B}(f)}$

where $P_{B}(f)$ is the Fourier power spectral density. These values are then normalised to the corresponding local magnetic field intensity averaged within each interval, $\langle B\rangle$, so that the $\delta B(f) /\langle B\rangle$ is a dimensionless quantity that can be compared in different solar wind regimes. According to the normalisation in Equation 4 , the Kolmogorov scaling $P_{B}(f) \sim f^{-5 / 3}$ corresponds to $\delta B(f) \sim f^{-1 / 3}$, and the Kraichnan scaling $P_{B}(f) \sim f^{-3 / 2}$ to $\delta B(f) \sim f^{-1 / 4}$, indicated in the figure as dashed lines. The lowfrequency part of the spectrum shows a flattening corresponding to the $1 / f$ scaling, indicating that the amplitude of the Fourier modes has saturated as discussed in Matteini et al. (2018) and 


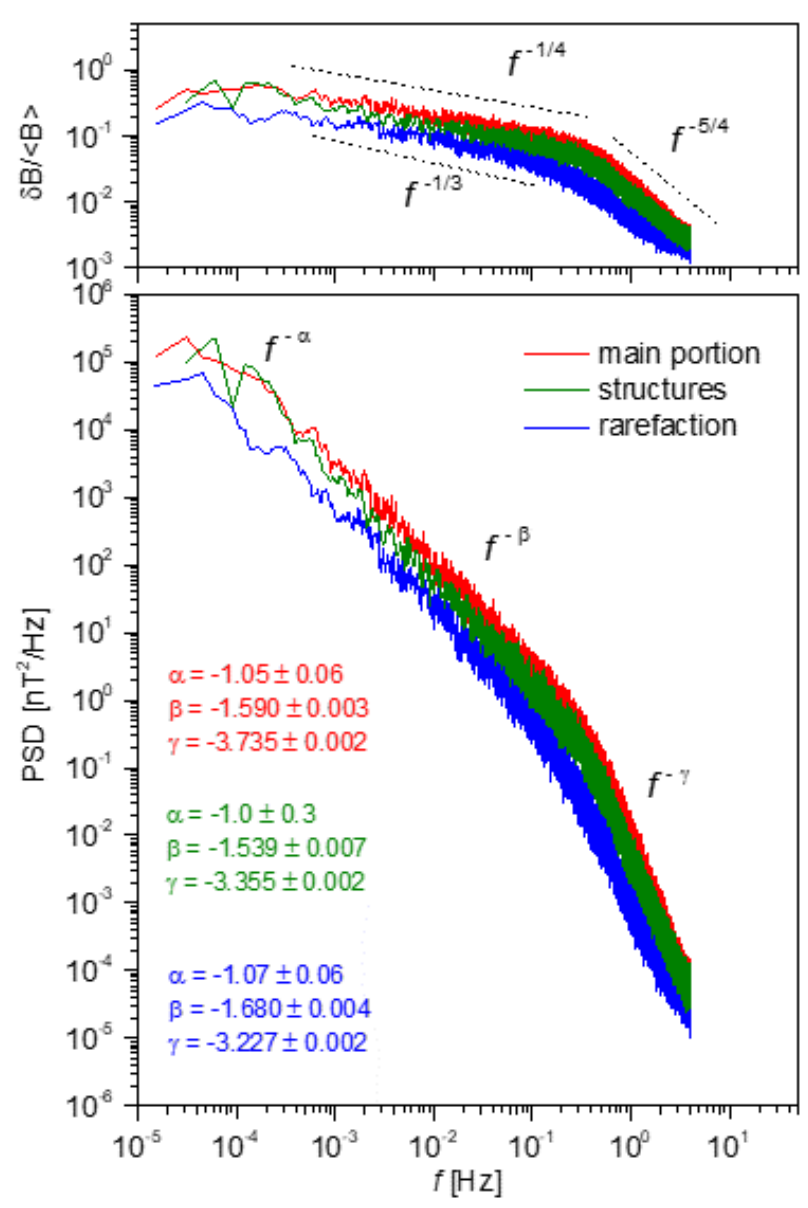

Fig. 6. Top panel: Normalised Power Spectral Density (PSD), $\delta B /\langle B\rangle$, derived as explained in the text, for the main portion of the stream (red), for the rarefaction region (blue), and for the intermediate region (green). The dashed lines correspond to the Kolmogorov scaling $\left(\delta B(f) \sim f^{-1 / 3}\right)$ and to the Kraichnan scaling $\left(\delta B(f) \sim f^{-1 / 4}\right)$, along with a scaling $\delta B(f) \sim f^{-5 / 4}$ corresponding to $P(f) \sim f^{-7 / 2}$, according to the normalisation described in the text. Bottom panel: PSD of the trace of magnetic field components for the three identified regions as in the top panel. The slopes of the three frequency regimes are indicated with the same color code of the different portions of the stream and are computed over the following frequency ranges: $f<3 \cdot 10^{-4} \mathrm{~Hz}, 2 \cdot 10^{-3}<f<10^{-1} \mathrm{~Hz}$ and $2 \cdot 10^{-1}<f<2 \mathrm{~Hz}$.

Bruno et al. (2019). The normalised PSD clearly shows that the three regions have different relative fluctuations, with the main portion of the stream characterised by the largest relative fluctuations respect to the average background magnetic field than the other two regions. Indeed, the relative fluctuations decrease as one moves from the main portion to the rarefaction region, with the region characterised by the presence of the structures having an intermediate value.

\subsubsection{Magnetic helicity spectrograms}

To better characterise the different portions of the stream, we use a measure of the magnetic helicity, $H_{m}$, an invariant of ideal magnetohydrodynamics (MHD), to analyze the nature of the magnetic field fluctuations using their polarisation properties. Matthaeus et al. (1982) first introduced the fluctuating reduced magnetic helicity for single-spacecraft observations, which gives the degree and handedness of helical rotations in B at a given frequency. More recently, this definition has been ex- tended to wavelet analysis (e.g. Telloni et al. 2012), and further refined to separate helicity contributions from different fluctuations (Woodham et al. 2021). For this part of the analysis, we use $64 \mathrm{~Hz}$ burst-mode magnetic field measurements from the MAG instrument, which has continuous coverage during our interval.

We calculate the normalised magnetic helicity in RTN coordinates averaged over 1-min intervals:

$$
\sigma_{m}(t, f)=\frac{2 \mathfrak{J}\left\{\mathcal{W}_{T}^{*}(t, f) \mathcal{W}_{N}(t, f)\right\}}{\left|\mathcal{W}_{R}(t, f)\right|^{2}+\left|\mathcal{W}_{T}(t, f)\right|^{2}+\left|\mathcal{W}_{N}(t, f)\right|^{2}},
$$

where $\mathcal{W}_{i}(t, f)$ are the continuous Morlet wavelet transforms (Torrence \& Compo 1998) of the RTN magnetic field components. This averaging procedure helps to smooth out some of the variability in the full-resolution transform. We neglect the spectrum in that interval where a data gap is present within the corresponding 1-min interval. $\sigma_{m}$ takes values in the interval $[-1,1]$, where $\sigma_{m}=-1$ indicates fluctuations with purely left-handed helicity and $\sigma_{m}=+1$ purely right-handed helicity in the plasma frame. A value of $\sigma_{m}=0$ indicates no overall coherence.

In Figure 7, we plot $\sigma_{m}(t, f)$ for the interval 14.0-18.5 July. In the spacecraft frame, the sign of $\sigma_{m}$ depends on both the background field direction, $\mathbf{B}_{0}$ and the wave-vector $\mathbf{k}$ of the fluctuations, which is Doppler shifted due to the Taylor hypothesis (e.g. Woodham et al. 2019). Due to the small amplitude of the turbulent fluctuations at small scales, the MAG noise floor can lead to an artificial flattening of the power spectrum, at frequencies above about $1 \mathrm{~Hz}$ in our interval. As the signal-to-noise ratio decreases with increasing frequency, $\sigma_{m}$ decreases towards zero and so this signature is not real. We also see the presence of right-handed fluctuations at frequencies, $f>10 \mathrm{~Hz}$, which are associated with an increase in power above the MAG noise floor. The Solar Orbiter Radio and Plasma Waves (RPW) (Maksimovic et al. 2020) observations by the search coil magnetometer are able to properly characterise these wave modes, which have been observed throughout the inner heliosphere (e.g. Lacombe et al. 2014; Jagarlamudi et al. 2020). We do not address these signatures further in this paper.

Fig. 7 shows that coherent magnetic helicity signatures are more prevalent in the main portion of the stream rather than in the intermediate region. Their occurrence rate in the rarefaction region is even lower. The magnetic helicity signatures at proton scales in Figure 7 are consistent with many previous studies throughout the heliosphere (He et al. 2011, 2012a,b; Podesta \& Gary 2011; Klein et al. 2014; Bruno \& Telloni 2015; Telloni et al. 2015; Telloni \& Bruno 2016; Telloni et al. 2019, 2020; Woodham et al. 2019, 2021). The right-handed signature of $\sigma_{m} \simeq 0.3$ centred at $\sim 1 \mathrm{~Hz}$ (close to the proton gyro-frequency, $f_{c p}$, shown as a solid black line in Fig. 7) is associated with right-handed turbulent fluctuations propagating anti-sunward, with polarisation properties consistent with KAWs (e.g., Howes \& Quataert 2010; He et al. 2012b). The helicity signatures $\sigma_{m} \simeq \pm 0.8$ between 0.1 $1 \mathrm{~Hz}$ are associated with small-scale kinetic instabilities driven by non-equilibrium features in the particle distribution functions (Kasper et al. 2002, 2008, 2013; Hellinger et al. 2006; Matteini et al. 2007; Bale et al. 2009; Maruca et al. 2012; Bourouaine et al. 2013; Gary et al. 2015; Alterman et al. 2018; Klein et al. 2018, 2019).

The sign of the helicity is dependent on the direction of propagation, which is not possible to determine due to the Doppler shift of proton distribution fucntions (Podesta \& Gary 2011; Woodham et al. 2019, 2021; Verniero et al. 2020; Bowen et al. 2020a; Bowen et al. 2020b). For an inward-oriented (negative 


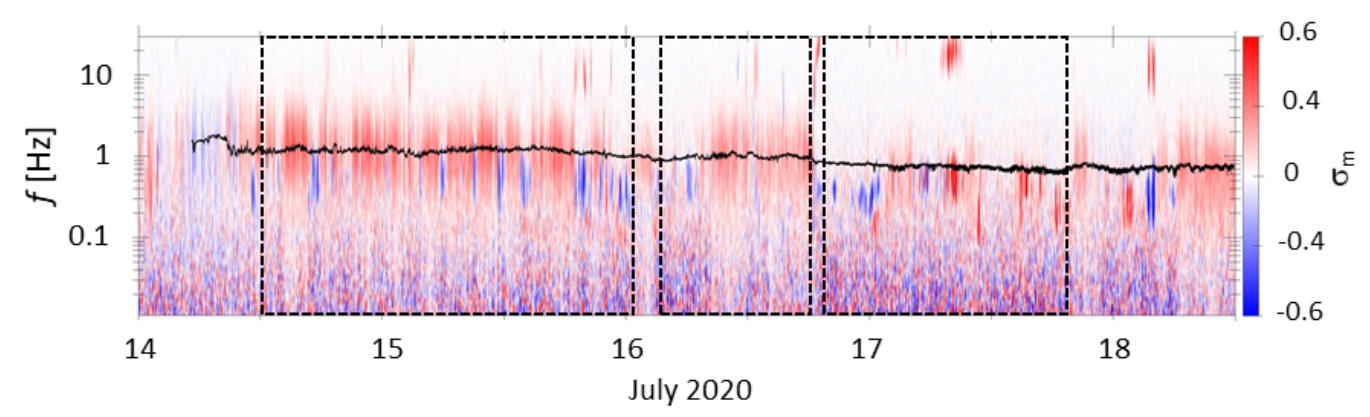

Fig. 7. Spectrogram of the normalised magnetic helicity, $\sigma_{m}$. The dashed boxes are the same identified in Fig. 4 . The black line corresponds to the proton gyro-frequency.

polarity) magnetic field, assuming outward propagation, a lefthanded ion-cyclotron wave would have a positive magnetic helicity and would correspond to a clockwise rotation of the magnetic field vector. The same wave would result in negative helicity for an outward-oriented magnetic field (Narita et al. 2009; He et al. 2011). On the contrary, a right-handed kinetic Alfvén wave would have a negative magnetic helicity and would correspond to an anti-clockwise rotation of the magnetic field vector for an inward magnetic field and would have a positive magnetic helicity for an outward magnetic field. Hence, it is important to evaluate the angle $\theta_{B R}$ between the sampling direction assumed along the radial direction and the scale-dependent mean magnetic field. For this purpose, a complementary approach follows the angular distribution of the magnetic helicity. Based on Horbury et al. (2008), $\theta_{B R}$ between the local mean magnetic field and the sampling direction, is first computed as a function of $t$ and $f$. Then, $\sigma_{m}(t, f)$ is reordered into $\sigma_{m}\left(\theta_{B R}, f\right)$, as first reported in literature by Horbury et al. (2008); He et al. (2011); Podesta \& Gary (2011) and later on by Bruno \& Telloni (2015); Telloni et al. (2015, 2020). For computational reasons, we use here normal mode MAG data at $16 \mathrm{~Hz}$.

The character of the fluctuations beyond the high-frequency break located between the fluid and kinetic regimes strongly depends on the Alfvénic content of the fluctuations in the inertial range and on their amplitude (Telloni \& Bruno 2016; Telloni et al. 2019; Woodham et al. 2021). Similar to the fast wind (Bruno \& Telloni 2015) and to a previous study on the Alfvénic slow wind at 1 AU (Telloni et al. 2020), in the present study the main portion of the stream shows a clear signature of both right-handed and left-handed polarised fluctuations, around $90^{\circ}$ and $0^{\circ}$, possibly associated with quasi-perpendicular KAWs and quasi-parallel ICWs, respectively (see Fig. 8, upper panel). On the other hand, the intermediate region and the rarefaction region (intermediate and lower panel), where the wind speed and the Alfvénicity of low-frequency fluctuations decrease, show a reduction in the presence of the ICW signature, in agreement with Bruno \& Telloni (2015). It is worth noticing that $\theta_{B R}$ in the rarefaction region is limited to values smaller than $90^{\circ}$ as also observed in Fig. 4.

\subsection{Magnetic structures}

Fig. 4 clearly show that this stream can be divided in welldefined regions, each one with peculiar characteristics. While the previous sections were devoted to a global comparative study of the three sub-intervals, in this section we focus on the characterization of the region marked by a dashed green box in Fig. 4 (16.2-16.9 July).
Fig. 9 shows the time series of plasma and magnetic field parameters for this region: the solar wind speed, $V_{s w}$ (panel a); the normal, $N$, components of velocity, $V_{N}$, and magnetic field in Alfvén units, $V_{A N}$ in RTN coordinate system (b); the proton number density, $n_{p}$ (c), the proton temperature, $T_{p}(\mathrm{~d})$, along with magnetic field magnitude (e), azimuthal, $\Phi_{B}$ (f) and polar angles, $\Theta_{B}(\mathrm{~g})$ and $\theta_{B R}(\mathrm{~h})$. The bottom panels display kinetic, $p_{k}$, magnetic, $p_{m}$ and total pressure, $p_{\text {tot }}$ (i) and the plasma beta, $\beta$ (l). These parameters allow us to identify different plasma parcels within the stream lasting typically around 30 minutes.

The last two structures at the end of the interval, highlighted with light red (labeled $\mathcal{B}_{0}$ ) and light blue (labeled $\mathcal{R}$ ) boxes, respectively, last longer. $\mathcal{B}_{0}$ and $\mathcal{R}$ are separated by the reconnection exhaust crossing discussed in Sec. 3.2 event. The structure indicated as $\mathcal{R}$ has been already identified as the beginning of the rarefaction region. Although the magnetic field magnitude is almost the same, $\mathcal{B}_{0}$ and $\mathcal{R}$ are oriented in two different directions (compare the magnetic field azimuthal and polar angles, $\Phi_{B}$ and $\Theta_{B}$, and $\theta_{B R}$ in Table 1) and also the plasma parameters identify different plasma parcels (notice the different but almost constant values of $n_{p}$ and $T_{p}$ within the two structures). On the other hand, the behaviour of the pressures is similar in the two cases, with a similar behaviour for $p_{k}$ and $p_{m}$, so that the plasma $\beta$ is similar and smaller than 1 . Not least, $\mathcal{B}_{0}$ is quite Alfvénic while in $\mathcal{R}$ the $v-b$ correlations are reduced (see also the behaviour of $\sigma_{c}$ in Fig. 2 and of $\rho_{v b}$ in Fig. 4).

Several structures at different scales are observed recalling the spaghetti-like flux-tube texture of the interplanetary magnetic field (McCracken \& Ness 1966; Mariani et al. 1973; Neugebauer 1981; Bruno et al. 2001; Borovsky 2008). The idea is that the $\mathrm{s} / \mathrm{c}$ might cross the same structure during successive time intervals. To prove this hypothesis, $\mathcal{B}_{0}$ along with the other parcels identified as $\mathcal{B}_{i}(\mathrm{i}=1,2,3)$ were gathered in a group as they display similar temporal evolution of different parameters. In a similar way, parcels labeled $\mathcal{A}_{i}$ were organized in a different group. In general, $\mathcal{B}$ structures show higher $n_{p}$ and lower $T_{p}$ values than $\mathcal{A}$ structures. Moreover, Table 1 clearly shows that the structures in each group have a different magnetic field orientation. In addition, most of the structures are quite Alfvénic (except for the subinterval 16.5-16.6 comprising the magnetic dip and other portions not highlighted in the boxes) and are generally separated from one another by sharp discontinuities similar to the tangential discontinuities already highlighted in previous studies (e.g. Bruno et al. 2001). Indeed, along all the interval 16.2-16.9, there is no large variation of pressure values except for the dip in the magnetic field around 16.5 that involves structures $\mathcal{A}_{1}, \mathcal{A}_{2}$ and $\mathcal{B}_{1}$. In this case, $p_{k}>p_{m}$ thus resulting in a $\beta$ much greater than 1. A similar behaviour is observed in the reconnection event. In the rest of the interval $p_{m}>p_{k}$ and $\beta<1$. 

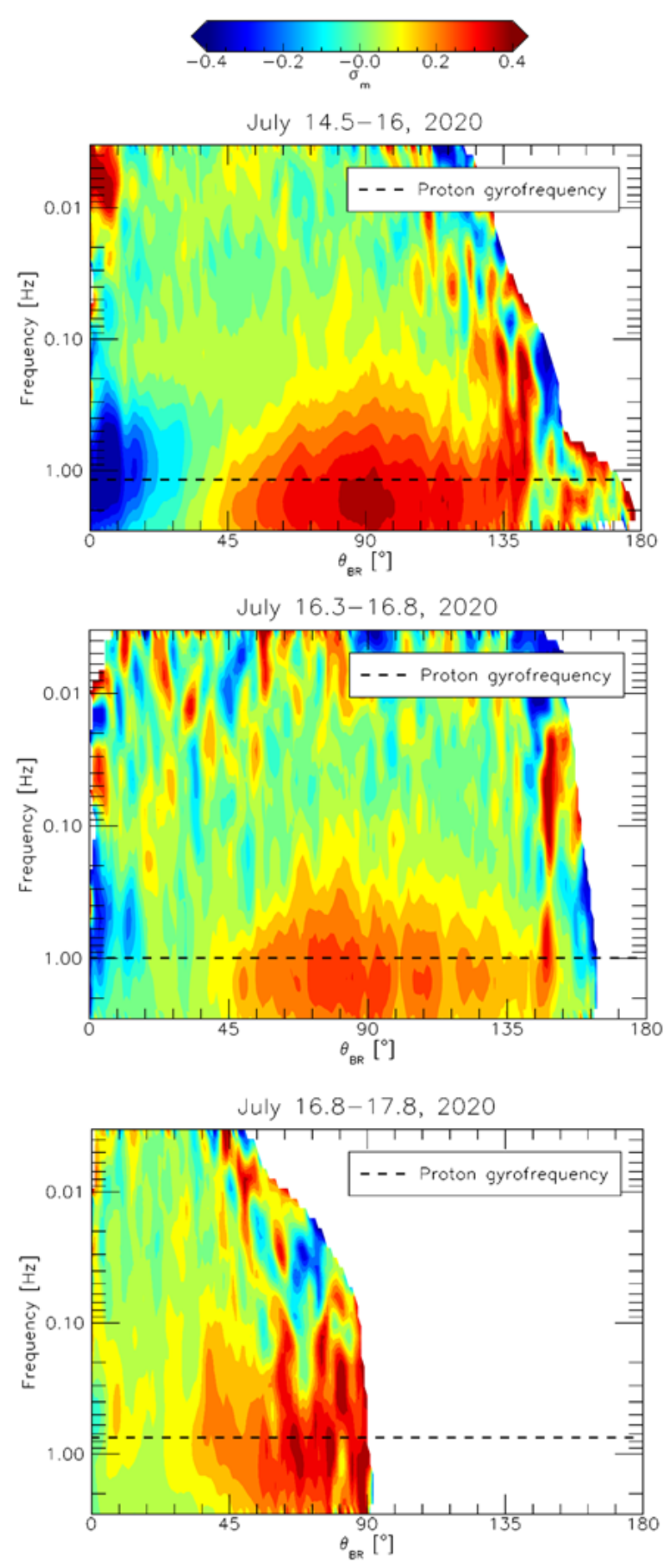

Fig. 8. Scalogram of the normalised magnetic helicity, $\sigma_{m}$ (color map), with respect to the angle, $\theta_{B R}$, between the magnetic field and the radial direction. From top to bottom: main portion of the stream, intermediate region and rarefaction region. The dashed line in each plot corresponds to the proton gyrofrequency in the $\mathrm{s} / \mathrm{c}$ frame.

In particular, $\beta \sim 0.7$, before the dip, while $\beta$ is close to 0.5 after the dip.

A MV analysis further reveals the intrinsic similarities and differences of the two groups and of the rarefaction region. The maximum, intermediate and minimum eingenvalues of the MV matrix are identified as $\lambda_{\text {max }}, \lambda_{\text {int }}, \lambda_{\text {min }}$ respectively. In all cases, $\lambda_{\min } \ll \lambda_{\max }, \lambda_{\text {int }}$ thus clearly identifying the MV direction by

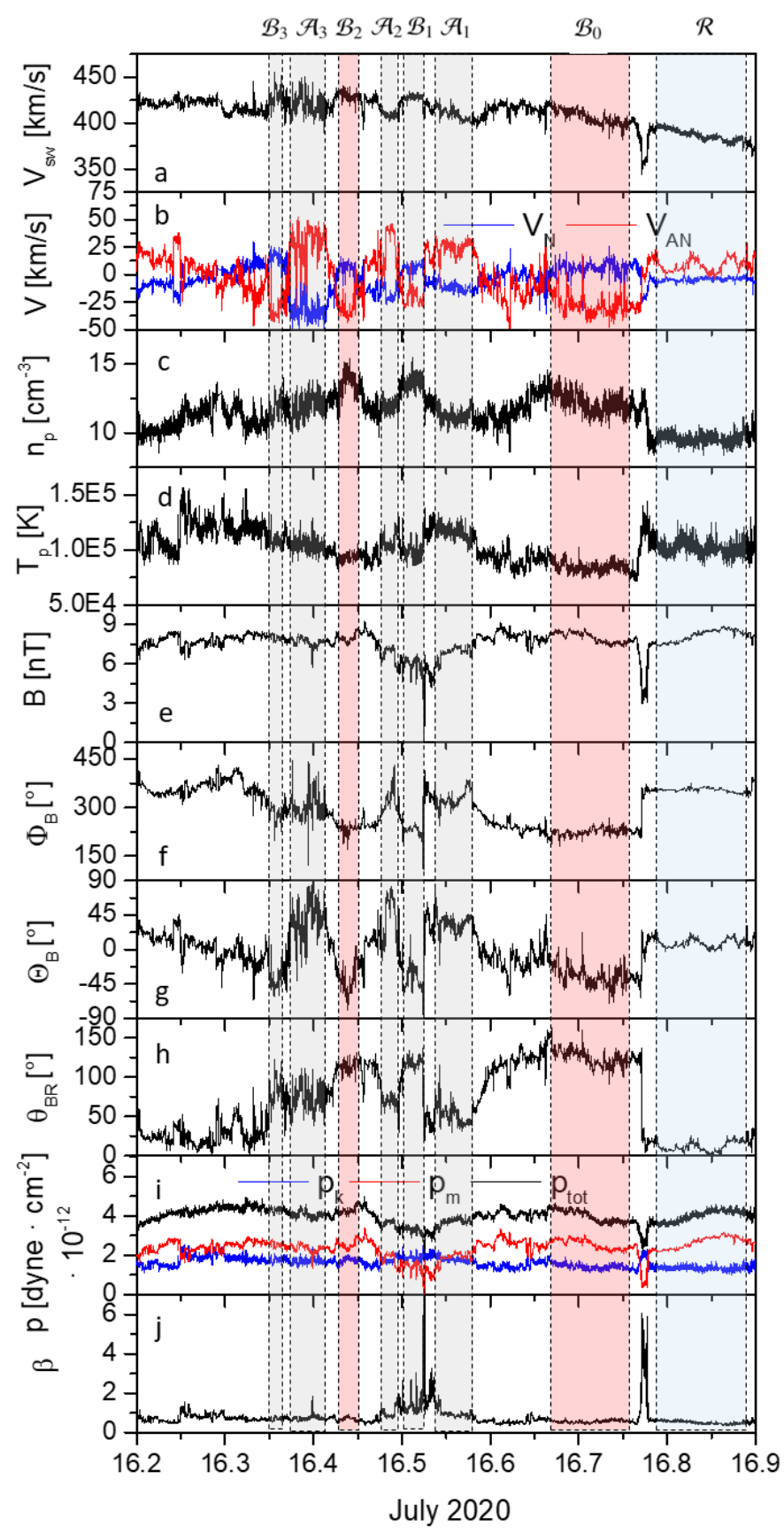

Fig. 9. Time series of relevant parameters corresponding to the interval 16.2-16.8. From top to bottom: the solar wind speed, $V_{s w}$ (panel a); the $\mathrm{N}$ components of velocity, $V_{N}$, and magnetic field in Alfvén units, $V_{A N}$ (b); the proton number density, $n_{p}$ (c), the proton temperature, $T_{p}(\mathrm{~d})$, along with magnetic field magnitude (e), azimuthal, $\Phi_{B}$ (f) and polar angles, $\Theta_{B}(\mathrm{~g})$ and $\theta_{B R}(\mathrm{~h})$; kinetic, $p_{k}$, magnetic, $p_{m}$ and total pressure, $p_{\text {tot }}$ (i) and the plasma beta, $\beta(\mathrm{j})$. The color boxes identify the structures which have been grouped in structures $\mathcal{A}$ and $\mathcal{B}$, along with the beginning of the rarefaction region identified as $\mathcal{R}$. All the structures have been identified with grey boxes apart from $\mathcal{R}$ in light blue and $\mathcal{B}_{0}$ and $\mathcal{B}_{2}$ with light red as explained in the text.

means of the angle the MV forms with the average magnetic field $\left(\theta_{B-M V}\right)$ and with the radial direction $\left(\theta_{R-M V}\right)$. Table 1 contains a summary of the MV variance analysis performed over all the structures labeled $\mathcal{A}_{i}$ and $\mathcal{B}_{i}$ and also on $\mathcal{R}$. From the comparison of magnetic field orientation and of the MV results, we can easily exclude $\mathcal{B}_{1}$ from 'group $\mathcal{B}$ '. Actually, although the magnetic 
field magnitude is almost constant along the whole interval 16.216.9 , there is a large depression of the field from 16.45 to 16.6 which results in $p_{m}$ lower than $p_{k}$ and consequently in $\beta>1$, suggesting that this plasma portion is different from $\mathcal{B}_{0}, \mathcal{B}_{2}$ and $\mathcal{B}_{3}$. Table 1 also shows that, although the MV analysis of structure $\mathcal{B}_{3}$ returns $\theta_{B-M V}$ and $\theta_{R-M V}$ similar to those of structures $\mathcal{B}_{0}$ and $\mathcal{B}_{2}$, the orientation of the large-scale magnetic field is different, leading to exclude also structure $\mathcal{B}_{3}$ from group $\mathcal{B}$.

Regarding group $\mathcal{A}$, a first important comment concerns the Alfvénic content of the fluctuations of these flux tubes. While $\mathcal{A}_{3}$ is quite Alfvénic, in $\mathcal{A}_{1}$ and $\mathcal{A}_{2}$, identified within a magnetic dip, $v-b$ correlations are almost absent. Moreover, when comparing the MV analysis (see Table 1 ) for $\mathcal{A}_{1}$ and $\mathcal{A}_{2}$, we end up with completely different MV directions, thus suggesting that these structures are not the same flux tube observed at different time intervals.

The identification of the previous structures might be an evidence of the spaghetti-like structure of the interplanetary magnetic field, which probably has its origin at the base of the solar atmosphere (e.g. Bruno et al. 2001; Borovsky 2008). Within this context, the two main candidates to be identified as the same structure crossed by the s/c in two different intervals are structures $\mathcal{B}_{0}$ and $\mathcal{B}_{2}$ as also shown in the $3 \mathrm{D}$ representation of the magnetic field components in RTN coordinate system of Fig. 10 (left panel), in grey and black dots, respectively. Indeed, they identify similar arc-like structures, moving on a sphere. The good correlation between magnetic and velocity fluctuations for both time intervals highlights the presence of Alfvénic fluctuations. In both cases, these fluctuations lie in a plane almost perpendicular to the average field direction since the angle between this direction and the MV direction is about 6-7 $7^{\circ}$ The results of this analysis further support the idea by Bruno et al. (2001) of an interplanetary magnetic field consisting of a bunch of flux tubes convected by the wind, which are entangled in space. According to this view, in each flux tube the presence of Alfvénic fluctuations makes the magnetic field vector randomly wander about a local field direction. The border between these flux tubes can be a tangential discontinuity where the total pressure on both sides of the discontinuity is in equilibrium or, in other cases, the discontinuity is located between two regions not in pressure equilibrium. An example of a tangential discontinuity is shown in Fig. 10 (right panel) which occurred on July 16.422 (just preceding structure $\mathcal{B}_{2}$ ). We selected a time interval of about 4 min of magnetic field data centered around the discontinuity and performed the MV analysis. The data in the left panel of Fig. 10 are rotated in the MV system. The associated abrupt change of the magnetic field direction is clearly visible in the plane containing the maximum and intermediate variance directions. From the MV analysis, we established the normal direction to the discontinuity, coincident with the MV direction which is almost aligned with the average magnetic field direction, $\left(\theta_{B-M V}=2.7^{\circ}\right)$. Moreover, in the RTN coordinate system the MV direction forms an angle $\theta_{M V}=2^{\circ}$ and $\phi_{M V}=277^{\circ}$, indicating the polar and azimuthal angles respectively. Therefore, the MV direction lies at a small angle from the $R T$ plane and it is quasi-parallel to the $T$ direction.

The interpretation of this structures according to the spaghetti model is based on the idea that solar wind fluctuations are a superposition of propagating Alfvén waves and convected structures (Bavassano \& Bruno 1989) that might be pressure balance structures as firstly pointed out by Tu \& Marsch (1990, 1993).

\section{Solar sources}

Following the solar wind and magnetic field in situ observations presented in Fig. 11 (top panels), Solar Orbiter's magnetic connectivity to solar sources was investigated for the time interval 14 - 17 July 2020. To this end, a Potential Field Source Surface (PFSS) model developed by Schrijver \& De Rosa (2003) was used. The PFSS model provides a qualitatively reliable model of the overall topology of the magnetic field below the source surface (SS), a surface at which all magnetic field lines are assumed to be open and radial. A detailed description of the method can be found in Panasenco et al. (2020). The solar wind streams seen by Solar Orbiter were traced down towards their source regions on the Sun, to a height of about $1.16 R_{\odot}$ (not directly to the photospheric level to reduce the topological noise), using the field lines obtained via PFSS extrapolation with different SS heights and the position of the Solar Orbiter extrapolated field line intersection on the SS. In order to trace the latter, a ballistic extrapolation from Solar Orbiter inward using the wind speed measured at Solar Orbiter down to the SS height was carried out. To analyze the robustness of such source identification with respect to wind acceleration profiles, corrections of up to $\pm 80 \mathrm{~km} \mathrm{~s}^{-1}$, in bins of $10 \mathrm{~km} \mathrm{~s}^{-1}$ were incorporated for the wind speed; these are added/subtracted from the measured solar wind velocity, and the ballistic extrapolation is repeated for the speeds measured over the four intervals as shown in Fig. 11. For the given time interval, the source surface height was determined for the specific regions by comparing the magnetic polarity of the obtained solar wind sources with the ones measured in situ. As shown in Panasenco et al. (2020), the source surface height $\left(R_{S S}\right)$ changes significantly on an hourly basis, especially during minimum of the solar activity, when the global magnetic field is fairly weak overall and, therefore, cannot inflate the SS to its typical height of about $2.5 R_{\odot}$ (Altschuler \& Newkirk 1969).

As the result of the mapped magnetic connectivity, Solar Orbiter's in situ wind characteristics were found to depend on a list of factors deduced from the coronal and photospheric observations of the corresponding wind source regions: streamers or pseudostreamers; coronal hole center or boundaries; presence or absence of filaments in the pseudostreamer lobes; monotonic vs non-monotonic expansion of the open field in the source region; phase of the solar activity. Concerning the last point, July 2020 was an extremely peculiar interval, since two active regions from two different solar cycles were present at the same period of time - an old cycle 24 active region (near the equator) and a new cycle 25 active region (mid latitudes).

For the magnetic and plasma properties observed by Solar Orbiter during the interval from 14-17 July, 2020 and shown in Fig. 11 (top panels), four periods can be identified with gradual changes in solar wind speed from 400 up to $450 \mathrm{~km} \mathrm{~s}^{-1}$ and then down to $320 \mathrm{~km} \mathrm{~s}^{-1}$. All, except one on July 16th, 18:04 UT, magnetic pressure maps and Solar Orbiter magnetic connections in Fig. 11 (bottom panels a-d) were calculated for $R_{S S}=2.0 R_{\odot}$ using high-resolution SDO/HMI (Solar Dynamics Observatory/Helioseismic and Magnetic Imager) magnetograms and evolving PFSS by Schrijver \& De Rosa (2003). The in-situ magnetic field displayed mostly a positive polarity, with a very brief connection to a small negative polarity area. Since there was no sharp density increase during the short polarity reversal on July 16 (Fig. 11 top panel, in situ plasma properties), it would appear that Solar Orbiter did not cross the combined heliospheric current/plasma sheet, perhaps only crossing the current sheet in and out as a result of current sheet folds (or thinning), and the complex coronal magnetic topology at the solar source. The shift 
Table 1. Magnetic field orientation and results of the Minimum Variance analysis of sub-intervals within July 16: group $\mathcal{B}$, group $\mathcal{A}$ and the rarefaction region, $\mathcal{R}$, respectively .

\begin{tabular}{c|cccc|ccc|c}
\hline \hline & $\mathcal{B}_{0}$ & $\mathcal{B}_{1}$ & $\mathcal{B}_{2}$ & $\mathcal{B}_{3}$ & $\mathcal{A}_{1}$ & $\mathcal{A}_{2}$ & $\mathcal{A}_{3}$ & $\mathcal{R}$ \\
\hline$\Phi_{B}$ & $230^{\circ}$ & $233^{\circ}$ & $237^{\circ}$ & $287^{\circ}$ & $294^{\circ}$ & $317^{\circ}$ & $329^{\circ}$ & $359^{\circ}$ \\
$\Theta_{B}$ & $-26.4^{\circ}$ & $-38.8^{\circ}$ & $-43.1^{\circ}$ & $-32.3^{\circ}$ & $41.2^{\circ}$ & $56.7^{\circ}$ & $35.8^{\circ}$ & $10.7^{\circ}$ \\
$\theta_{B R}$ & $125^{\circ}$ & $118^{\circ}$ & $114^{\circ}$ & $74.6^{\circ}$ & $72.1^{\circ}$ & $65.9^{\circ}$ & $45.9^{\circ}$ & $13.9^{\circ}$ \\
\hline$\lambda_{\text {max }}$ & 5.11 & 2.16 & 4.23 & 6.05 & 9.51 & 12.2 & 5.07 & 1.60 \\
$\lambda_{\text {int }}$ & 2.56 & 0.504 & 0.651 & 2.14 & 2.87 & 1.51 & 0.720 & 0.582 \\
$\lambda_{\text {min }}$ & 0.507 & 0.393 & 0.161 & 0.636 & 1.01 & 0.172 & 0.436 & 0.148 \\
$\lambda_{\text {int }} / \lambda_{\text {max }}$ & 0.500 & 0.234 & 0.154 & 0.353 & 0.301 & 0.123 & 0.142 & 0.363 \\
$\lambda_{\text {min }} / \lambda_{\text {max }}$ & 0.059 & 0.182 & 0.038 & 0.105 & 0.106 & 0.021 & 0.086 & 0.092 \\
$\lambda_{\text {min }} / \lambda_{\text {int }}$ & 0.158 & 0.780 & 0.248 & 0.297 & 0.353 & 0.172 & 0.605 & 0.254 \\
\hline$\theta_{B-M V}$ & $7.37^{\circ}$ & $64.5^{\circ}$ & $6.34^{\circ}$ & $3.65^{\circ}$ & $9.63^{\circ}$ & $66.8^{\circ}$ & $28.3^{\circ}$ & $3.33^{\circ}$ \\
$\theta_{R-M V}$ & $60.2^{\circ}$ & $4.29^{\circ}$ & $70.2^{\circ}$ & $73.7^{\circ}$ & $62.9^{\circ}$ & $4.51^{\circ}$ & $71.0^{\circ}$ & $13.0^{\circ}$ \\
\hline
\end{tabular}

Notes. From top: the average azimuth $\Phi_{B}$ and polar angles $\Theta_{B}$ of the magnetic field vector; the angle $B$ forms with the radial direction $\theta_{B R}$; the eigenvalues of the minimum variance matrix $\left(\lambda_{\min }, \lambda_{\text {int }}, \lambda_{\max }\right)$ along with their ratios $\left(\lambda_{\text {int }} / \lambda_{\max }, \lambda_{\min } / \lambda_{\max }, \lambda_{\min } / \lambda_{\text {int }}\right)$; the angle the minimum variance forms with the average magnetic field $\left(\theta_{B-M V}\right)$ and with the radial direction $\left(\theta_{R-M V}\right)$.
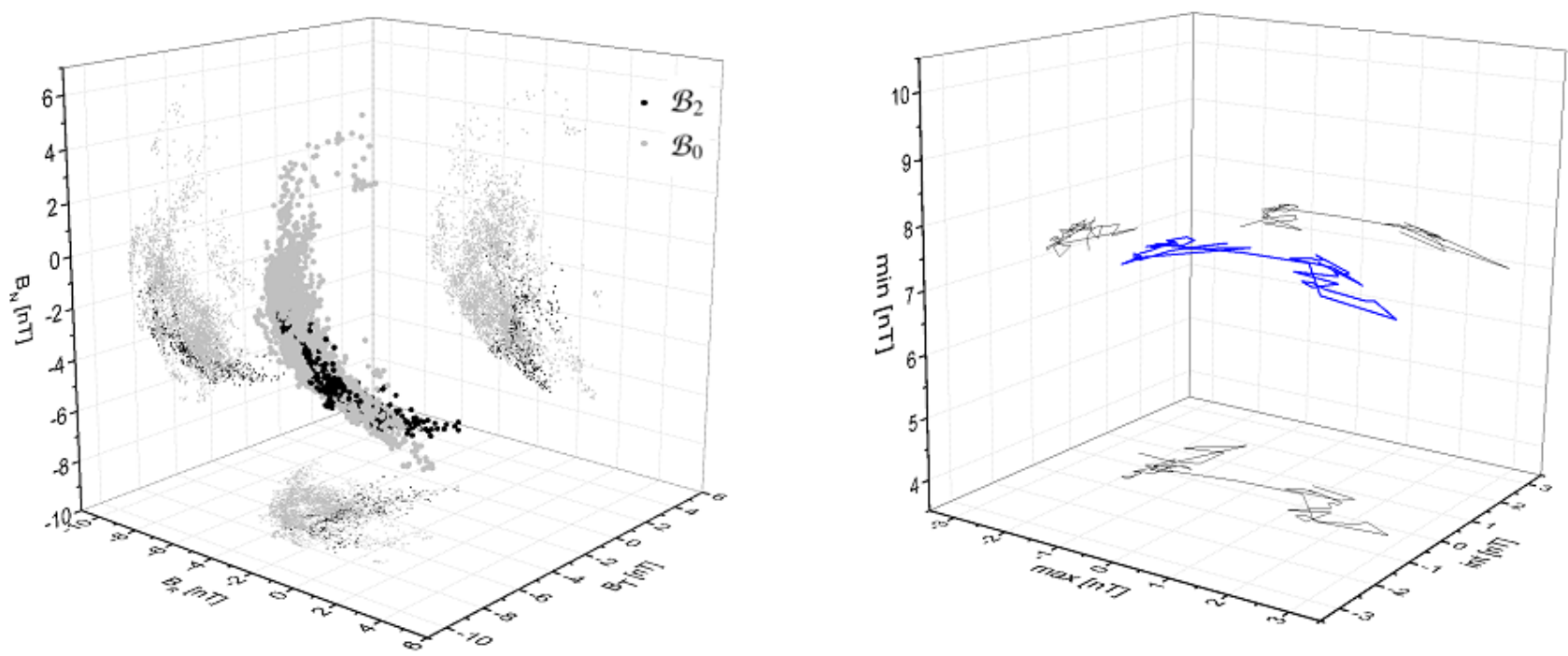

Fig. 10. Left: $3 \mathrm{D}$ representation of magnetic field components in $R T N$ coordinate system for the structures labeled $\mathcal{B}_{0}$ (grey dots) and $\mathcal{B}_{2}$ (black dots), along with their projections in the $R T, R N$ and $T N$ planes. Right: $3 \mathrm{D}$ representation of magnetic field components across a tangential discontinuity preceding structure $\mathcal{B}_{2}$ in the MV system, along with their projections in the maximum-minimum, maximum-intermediate and minimum-intermediate planes.

between the current and plasma sheet positions is a relatively normal phenomenon, and was observed by Ulysses (Winterhalter et al. 1994; Smith 2001). For comparison, the plasma properties measured before 12:00 UT on July 14th show a polarity reversal with a proper current and plasma sheet crossing indicated by the strong density increase, with prolonged decrease in the Alfvénicity. The origin of the negative open field area on 16 July was a magnetic flux imbalance in a decaying active region AR 12766 (part of the old cycle 24) that emerged near the equator on 3 July 2020 with a leading and stronger negative polarity. Ten days later this negative polarity attracted Solar Orbiter magnetic footpoints for a few hours. In order to correctly capture this polarity reversal using the PFSS and ballistic extrapolation methods, the SS height had to be brought down to $R_{S S}=1.7 R_{\odot}$ (Fig. 11, bottom panel c).

From PFSS $B^{2}$ contour maps and solar wind magnetic footpoints along the Solar Orbiter trajectory presented in Fig. 11 one can see gradual changes in the magnetic connectivity - from the internal part of the northern polar coronal hole extension (panel a) to its eastern boundary (panel b), to a short connection to the negative open field area at the equator (panel c), to the double coronal hole connections separated by a pseudostreamer (panel d). Topologically, this evolution in the Solar Orbiter connectivity goes from crossing a classic coronal helmet streamer to a pseudostreamer, two large-scale coronal configurations with intrinsically different properties reflected in the properties of the corresponding solar wind streams. In particular, in panel d, before July 18, though the wind is fairly Alfvénic, the amplitude of the radial magnetic field fluctuations and the corresponding "switchback patches" are smaller, and there are less of them, while on July 18, which corresponds to the traversal of Solar Orbiter's connection to the other side of the pseudostreamer, we see larger amplitude radial magnetic field fluctuations and a larger amplitude switchback "patch". This may be due to a change in the distance of the source to structures such as nearby neutral lines, presence or absence of active regions and filament channels.

Fig. 12 presents 3D PFSS rendering of the magnetic field lines of these streamer and pseudostreamer configurations. Both 

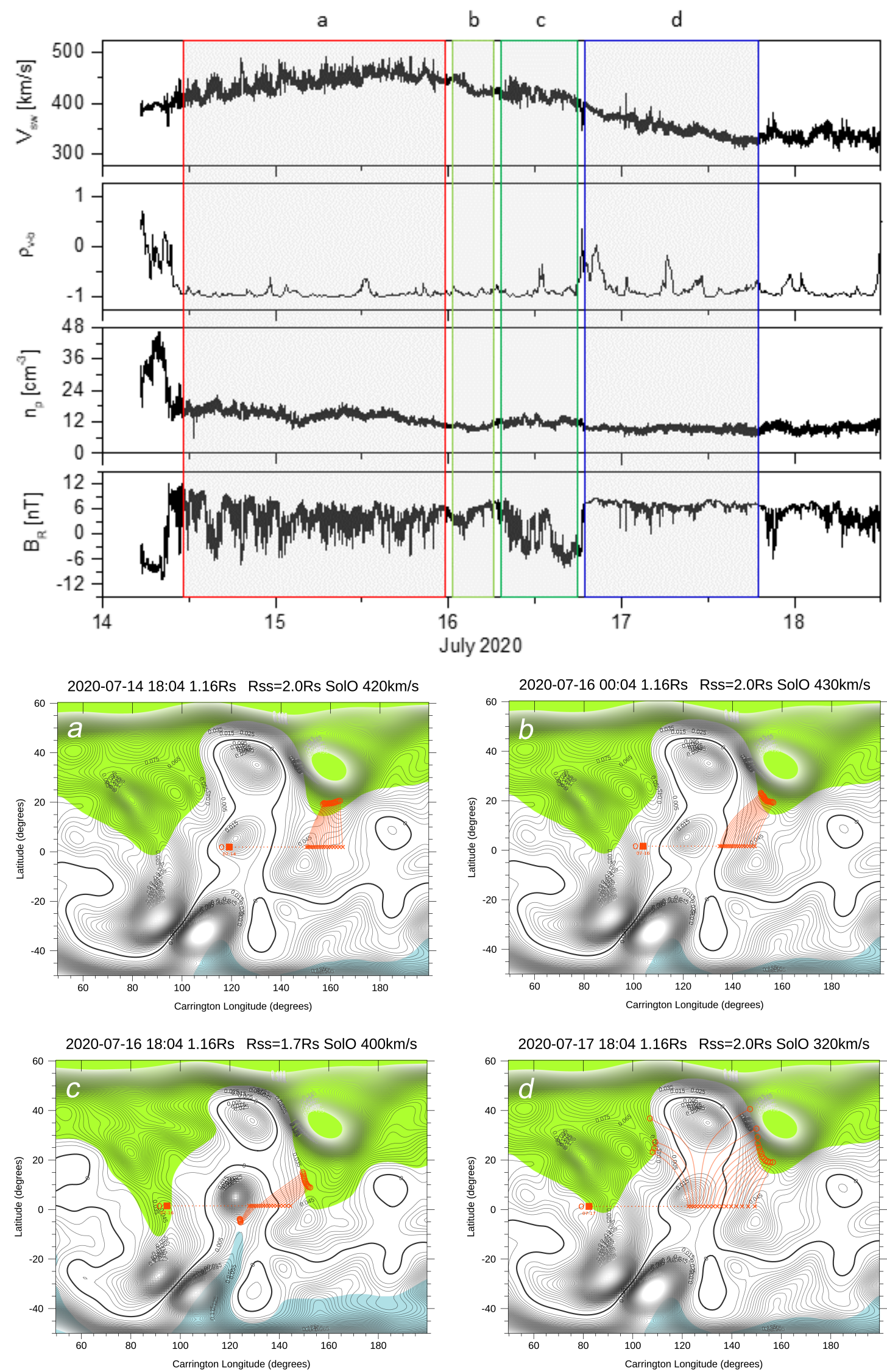

Fig. 11. Top: in situ plasma parameters; Solar Orbiter solar wind speed, $V_{s w}$ (in km/s); v-b correlation coefficient, $\rho_{v b}$; proton density (in $\mathrm{cm}^{-3}$ ); radial magnetic field magnitude, B (in nT). Bottom - Source maps: PFSS $B^{2}$ contour maps and solar wind magnetic foot-points along the Solar Orbiter trajectory for the time intervals a-d selected in the upper panel. The projection of Solar Orbiter location (orange square) on the source surface (orange crosses) and down to the solar wind source region (orange circles) calculated for the height $R=1.16 R_{\odot}$ and measured in situ solar wind speed $\pm 80 \mathrm{~km} \mathrm{~s}^{-1}$ in bins of $10 \mathrm{~km} \mathrm{~s}^{-1}$. Open magnetic field regions shown in blue (negative) and green (positive), the neutral line is in black bold. 
were calculated for $R_{S S}=2.0 R_{\odot}$ using high-resolution SDO/HMI (Solar Dynamics Observatory/Helioseismic and Magnetic Imager) magnetograms and evolving PFSS by Schrijver \& De Rosa (2003). The 3D PFSS model of the helmet streamer in the left panel was made along the 161 degree Carrington Longitude (see Fig. 11, panel a). Corresponding to this time, 14 July 2020, the Solar Orbiter magnetic footpoints are indicated with a white arrow. The right panel of Figure 12 illustrates the Solar Orbiter magnetic connection to coronal holes separated by a pseudostreamer configuration in the norther hemisphere, formed by two neighboring extensions of the positive polar coronal hole. The 3D PFSS model of the pseudostreamer was made along the 26-27 degree Carrington Latitude (see Fig. 11, panel d). Corresponding to this time, 17 July 2020, the Solar Orbiter magnetic footpoints are indicated with a white arrow.

Changes in the solar wind properties observed by Solar Orbiter in situ appear to be the result of the gradual motion of the coronal footpoints of the solar wind field lines measured by Solar Orbiter, from the streamer to pseudostreamer magnetic topology. The apparent difference in the behaviour of the open magnetic field lines shown in Fig. 12 can be simply described as a monotonic (left panel) and strongly non-monotonic (right panel) expansion of the open magnetic flux. Field lines in the neighborhood of pseudostreamers often have such strongly non-monotonic magnetic field expansion that was previously shown to be associated with slow Alfvénic solar wind streams (Panasenco et al. 2019, 2020). The solar magnetic connections of the missions such as Parker Solar Probe and Solar Orbiter target the boundaries of the open magnetic regions and, as shown in Panasenco et al. (2019), these boundaries are defined by the magnetic field lines with strongest non-monotonic expansion factor when a pseudostreamer configuration is present. The same seems to be the case in Fig. 12. The centers or internal areas of coronal holes have nearly perfect radial fields with monotonic expansion, especially for helmet streamer type configurations separating northern from southern polar coronal holes. The open magnetic field lines in the neighborhood of pseudostreamer lobes, however, follow the topology of the closed magnetic field lines inside pseudostreamer lobes, often dictated by the presence of a filament channel with a strong horizontal (parallel to the solar surface) component of the magnetic field. Such sensitivity can be attributed to the relatively short (above the solar surface) pseudostreamer configuration with x-point often at or below $1.3 R_{\odot}$. As for the pseudostreamer shown in Fig. 12, it was already well established in the solar corona a few weeks before Solar Orbiter connected to it, and it was harboring two filament channels, as well as dynamic filaments that erupted and reformed before Solar Orbiter connections.

The topology of this pseudostreamer (right panel in Fig.12) was perfectly fit to allow the formation and development of both filament channels, and they dictated strong non-monotonic expansion of the open magnetic field lines from the pseudostreamer coronal hole boundaries as was shown in Panasenco \& Velli (2013). In the presence of filament channels, the open magnetic field of pseudostreamers follows the guidance of the strong horizontal component of the magnetic field inside filament channels in the same way as chromospheric fibrils and coronal cells (Sheeley et al. 2013; Wang 2013; Panasenco et al. 2013). That creates conditions for strong divergence of the PS open field, which together with the non-monotonic expansion low in the corona (below $1.3-1.4 R_{\odot}$ ) slowing down the fast solar wind, creating environment for the development of the Alfvénic slow solar wind.
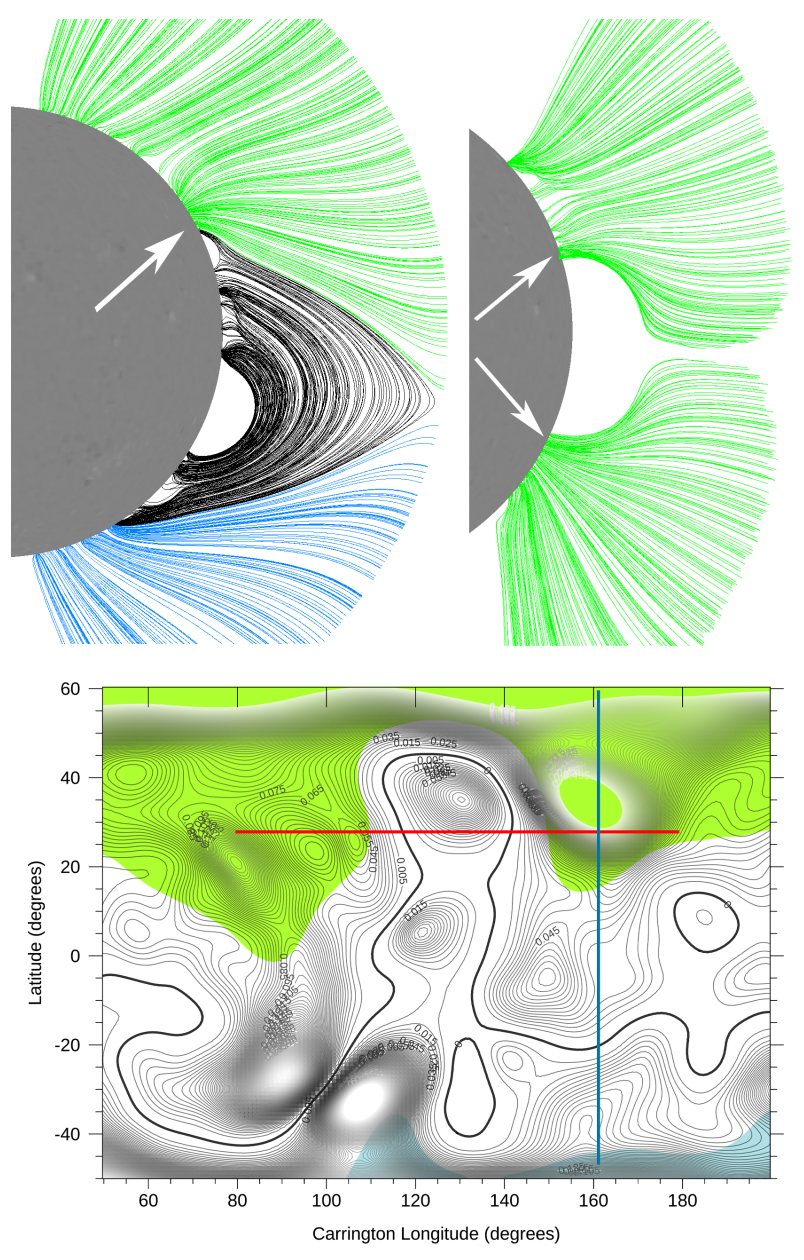

Fig. 12. Top left panel: solar magnetogram on disk with open magnetic field lines obtained via 3D PFSS modeling and $R_{S S}=2.0 R_{\odot}$ on 14 July 2020 18:04 UT using SDO/HMI data. The PFSS reconstructed magnetic field helmet streamer configuration is shown as it appears on the limb (corresponds to the map in Fig. 11a, the PFSS model was made along the 161 degree Carrington Longitude). Top right panel: 3D PFSS model for the pseudostreamer rotated to the limb view - an area of the origin of Alfvénic slow solar wind observed at Solar Orbiter on 17 July 2020 (corresponds to the map in Fig. 11d). White arrows point to the regions of the Solar Orbiter magnetic footpoints. Bottom panel: magnetic pressure map with locations of cuts along which the top panel 3D PFSS models were constructed. The blue cut corresponds to the top left, and the red cut to the top right model.

\section{Summary and discussion}

This study reports the first observation of an Alfvénic slow wind stream by Solar Orbiter at 0.64 AU during 14-18 July 2020. D'Amicis et al. (2021a) and references therein, highlighted several similarities of this solar wind regime to fast wind streams, spanning from the description of the large scale structure down to smaller scales. Indeed, the speed profile is similar to the fast wind (although with a lower speed) and consists of well-defined plasma regions, namely a compression region, the main portion of the stream and the rarefaction region. Solar Orbiter plasma and magnetic field measurements allowed to identify these regions. Since the compression region is partially missed, we focus on the main portion of the stream and on the rarefaction region. In particular, the former is characterised by large amplitude Alfvénic fluctuations, with a predominance of outward modes showing an energy imbalance in favor of magnetic en- 
ergy, in agreement with fast wind observations by Helios at the same heliocentric distance (Bruno et al. 2007). Related to a high Alfvénic content of the fluctuations is the presence of switchbacks which are the main features of this part of the stream. The presence of large amplitude Alfvénic fluctuations is also responsible for the presence of several magnetic helicity features identified as right-handed quasi-perpendicular KAWs and left-handed quasi-parallel ICWs at kinetic scales. Overall, the spectral features show PSD in accordance with the presence of Alfvénic fluctuations and which are well-described by three regimes (moving from low to high frequencies): an $1 / f$ scaling, a Kraichnan-like/Kolmogorov-like scaling and a steeper spectrum in the kinetic range. A different behaviour is observed in the rarefaction region. The main difference with the previous part of the stream is a sudden decay of v-b correlations with smaller amplitude of the fluctuations consistent with a reduced switchback activity, a lower amplitude power spectrum at all frequencies and the disappearance of magnetic helicity features around $1 \mathrm{~Hz}$.

A remarkable feature of this stream is the presence of several structures at different scales recalling the spaghetti-like flux-tube texture of the interplanetary magnetic field (McCracken \& Ness 1966; Mariani et al. 1973; Neugebauer 1981; Bruno et al. 2001; Borovsky 2008). In particular, our results suggest the possibility that the $\mathrm{s} / \mathrm{c}$ may cross the same structure during successive time intervals. Indeed, two structures with similar plasma parameters and magnetic field orientation have been identified, with the tip of the magnetic field moving on a sphere as expected for noncompressive fluctuations. Indeed, the fluctuations within these structures are strongly Alfvénic and lie in a plane almost perpendicular to the average field direction. The presence of these features are in agreement with the idea of solar wind fluctuations as a superposition of propagating Alfvén waves and convected structures (Bavassano \& Bruno 1989) which result in an interplanetary magnetic field consisting of a bunch of flux tubes convected by the wind and entangled in space (e.g. Bruno et al. 2001).

The magnetic connectivity of Solar Orbiter in situ observations to the solar sources during our interval was investigated using a PFSS model. In Fig. 11, we identified different intervals similar to the rest of the analysis. However, the intermediate region in Fig. 4 was split in two (see Fig. 11), discriminating the small negative polarity area on July 16 , that contrasts with the mostly positive polarity of the in-situ magnetic field throughout the rest of the stream. The origin of this negative open field area was a magnetic flux imbalance in a decaying equatorial active region AR 12766 related to the old solar cycle 24. PFSS magnetic pressure contour maps and solar wind magnetic foot-points along the Solar Orbiter trajectory presented in Fig. 11, highlight gradual changes in the magnetic connectivity - from the internal part of the northern polar coronal hole extension (panel a) to its eastern boundary (panel $b$ ), to a short connection to the negative open field area at the equator (panel c), to the double coronal hole connections separated by a pseudostreamer (panel d). The gradual motion of the coronal magnetic footpoints of the solar wind field lines measured by Solar Orbiter, from the coronal streamer to pseudostreamer magnetic topology determines changes of the solar wind properties observed by Solar Orbiter in situ. A region of anomalous expansion rate identified as the source of the Alfvénic slow wind (Panasenco et al. 2019, 2020; D'Amicis et al. 2021a, and references therein) may form easily when largescale pseudostreamers are present in the corona. The topology of pseudostreamers allows the formation and development of twin filament channels, related to a strong non-monotonic expansion of the open magnetic field lines from the PS coronal hole bound- aries as was shown in Panasenco \& Velli (2013); Panasenco et al. (2019). The presence of filament channels creates conditions for strong divergence of the pseudostreamer open field, which together with the non-monotonic expansion in the low corona (below $1.3-1.4 \mathrm{R}_{\odot}$ ) slow down the fast solar wind, allowing conditions for the development of the slow Alfvénic solar wind.

The observation of an Alfvénic slow wind stream by Solar Orbiter reported in this paper is extremely important to pioneer future observations of the same solar wind regime during maximum of solar activity, which is fast approaching, where this solar wind regime is predominant. At the same time, we are also approaching the nominal phase of the Solar Orbiter mission during which we will exploit the full potential of the mission which will combine in situ measurements and remote sensing observations, for the first time with a single spacecraft, in the inner heliosphere. Whether the slow solar wind originates from the over-expanded edges of coronal holes is one of the objectives of the Solar Orbiter Science Activity Plan (Zouganelis et al. 2020). The suggested strategy to answer this question is to exploit the remote-sensing windows to obtain a $\sim 3 \mathrm{D}$ view of the coronal hole edges using the Polarimetric and Helioseismic Imager, PHI (Solanki et al. 2020), and the Extreme Ultraviolet Imager, EUI (Rochus et al. 2020). Moreover, the METIS coronograph (Antonucci et al. 2020) will provide observations close to the Sun, while the Spectral Imaging of the Coronal Environment, SPICE (The SPICE Consortium 2020) will provide composition maps. SPICE will be used also to study coronal hole boundaries. These observations, along with SWA and MAG measurements, will allow unprecedented magnetic connectivity between the solar corona and the inner heliosphere. The SWA-HIS sensor deserves a particular mention as it will provide measurements of solar wind composition for the first time in the inner heliosphere providing additional data to establish the connection between the insitu observation of the Alfvénic slow solar wind with its source region.

Acknowledgements. Solar Orbiter is a space mission of international collaboration between ESA and NASA, operated by ESA. Solar Orbiter Solar Wind Analyser (SWA) data are derived from scientific sensors which have been designed and created, and are operated under funding provided in numerous contracts from the UK Space Agency (UKSA), the UK Science and Technology Facilities Council (STFC), the Agenzia Spaziale Italiana (ASI), the Centre National d'Etudes Spatiales (CNES, France), the Centre National de la Recherche Scientifique (CNRS, France), the Czech contribution to the ESA PRODEX programme and NASA. The AMDA science analysis system is provided by the Centre de Données de la Physique des Plasmas (CDPP) supported by CNRS, CNES, Observatoire de Paris and Université Paul Sabatier, Toulouse. CNES support for the PAS instrument embarked on Solar Orbiter is acknowledged by French co-authors. P.L. and A.F. thank E. Penou, A. Barthe and CDPP in preparing PAS/SWA data. Solar Orbiter magnetometer operations are funded by the UK Space Agency (grant ST/T001062/1). T.H. was supported by STFC grant ST/S000364/1. D.T. was partially supported by the Italian Space Agency (ASI) under contract I/013/12/0. M.V. acknowledges the support of the FIELDS experiment on the Parker Solar Probe spacecraft, designed and developed under NASA contract NNN06AA01C. O.Pa. was supported by the NASA grant 80NSSC20K1829. M.V. and O.Pa. acknowledge the support of the HERMES DRIVE NASA Science Center grant No. 80NSSC20K0604. R.D.M. was supported by the Italian Space Agency (ASI) under contract n. 2018-30-HH.O. L.S.V. was funded by the Swedish Contingency Agency grant 2016-2102 and by SNSA grant 86/20. D.V. was supported by STFC Ernest Rutherford Fellowship ST/P003826/1 and STFC Consolidated Grant ST/S000240/1. L.D.W. was supported by the STFC consolidated grant ST/S000364/1.

\section{References}

Alterman, B. L., Kasper, J. C., Stevens, M. L., \& Koval, A. 2018, APJ, 864, 112 Altschuler, M. D., \& Newkirk, G. 1969, Solar Physics, 9(1), 131 The SPICE Consortium 2020, A\&A, 642, A14

Antonucci, E., Romoli, M., Andretta, V. et al. 2020, A\&A, 642, A10 
Auchère, F., Andretta, V., Antonucci, E., et al. 2020, A\&A, 642, A6 Bale, S. D., Kasper, J. C., Howes, G. G., et al. 2009, PRL, 103, 211101 Bale, S. D., Badman, S. T., Bonnell, et al. 2019, Nature, 576(7786), 237 Balogh, A., Forsyth, R. J., Lucek, E. A., et al. 1999, GRL, 26(6), 631 Bavassano, B., \& Bruno, R. 1989, JGR, 94, 1168

Bavassano, B., Pietropaolo, E., \& Bruno, R. 1998, JGR, 103, 6521

Bavassano, B., Pietropaolo, E., \& Bruno, R. 2000, JGR ,105, 12697 Bavassano, B., \& Bruno, R. 2000, JGR, 105, 5113

Behannon, K. W., \& Burlaga, L. F. 1981, In Solar wind 4, 374

Belcher, J. W. \& Davis, L. 1971, JGRA, 76, 3534

Belcher, J. W. \& Solodyna, C. V. 1975, JGRA, 80, 181

Belcher, J.W., Davis, L. Jr., \& Smith, E.J. 1969, JGR, 74, 2302.

Borovsky, J. E. 2008, JGR, 113, A08110

Borovsky, J. E. 2016, JGR, 121, 5055

Bourouaine, S., Verscharen, D., Chandran, B. D., Maruca, B. A., \& Kasper, J. C. 2013, APJL, , 777, L3

Bowen, T. A., Mallet, A., Huang, J., et al. 2020a, APJS, 246, 66

Bowen, T. A., Bale, S. D., Bonnell, J. W., et al. 2020b, APJ, 899, 74

Bruno, R., Bavassano, B.,\& Villante, U. 1985, JGR, 90, 4373

Bruno, R., \& Bavassano, B. 1991, JGR, 96, 7841

Bruno, R., Carboneb, V.,Veltri, P., Pietropaolo, E., Bavassano, B. 2001, Planetary and Space Science, 49, 1201

Bruno, R., Carbone, V., Primavera, L., et al. 2004, AnnGeo, 22, 3751

Bruno, R., D’Amicis, R., Bavassano, B., et al. 2007, Annales Geophysicae, 25, 1913

Bruno, R., Trenchi, L., \& Telloni, D. 2014, The Astrophysical Journal Letters, 694793(1), L15

Bruno, R., \& Telloni, D. 2015, The Astrophysical Journal Letters, 811, L17

Bruno, R., \& Trenchi, L. 2014, Astrophys. J. 787, L24

Bruno, R., Telloni, D., Sorriso-Valvo, L., et al. 2019, A\&A, 627, A96

Bruno, R., \& Carbone, V. 2013, LRSP, 10, 2

Burlaga, L. F., \& Ogilvie, K. W. 1970, ApJ, 159, 659

Carnevale, G., Bruno, R., Marino, R. et al. 2021, A\&A, submitted

Chhiber, R., Goldstein, M. L., Maruca, B. A., et al. 2020, APJS, 246(2), 31

Coleman, P.J. 1968, APJ, 153, 371

D’Amicis, R., Bruno, R., \& Bavassano, B. 2007, GRL, 34, 5108

D’Amicis, R., Bruno, R., \& Bavassano, B. 2011, JASTP, 2011, 3, 653

D'Amicis, R., \& Bruno, R. 2015, APJ, 2015, 805, 84

D'Amicis, R., Matteini, L., \& Bruno, R. 2019, MNRAS, 483, 4665

D’Amicis, R., Matteini, L., Bruno, R., Velli, M. 2020, Solar Physics, 295, article id. 46

D’Amicis, R., Perrone, D., Velli, M., Bruno, R. 2021a, JGR, 126, e2020JA028996

D’Amicis, R., Alielden, K., Perrone, D., et al. 2021b, A\&A, submitted

Davis, M. S., Phan, T. D., Gosling, J. T., Skoug, R. M. 2006, GRL, 33, L19102

Del Zanna, L., Velli, M., \& Londrillo, P. 2001, A\&A, 367, 705

Dmitruk P., \& Matthaeus W. H., 2007, Phys. Rev. E, 76, 036305

Dudok de Wit, T., Krasnoselskikh, V. V., Bale, S. D. et al. 2020, APJS, 246(2), 39

Elliott, H. A., Henney, C. J., McComas, D. J., Smith, C. W., \& Vasquez, B. J. 2012, JGR, 117, A09102

Elsässer, W. M. 1950, Phys. Rev., 79, 183

García Marirrodriga, C., Pacros, A., Strandmoe, S., et al. 2021, A\&A, 6646, A121

Gary, S. P., Jian, L. K., Broiles, T. W., et al. 2015, JGR, 121, 30

Goelzer, M. L., Schwadron, N. A., \& Smith, C. W. 2014, JGRA, 119, 115

Goldstein, M. L., Roberts, D. A., \& Fitch, C. A. 1994, JGR, 99, A6, 11519

Grappin, R., Velli, M., \& Mangeney, A. 1991, Ann. Geophys., 1991, 9, 416

Hamilton, K., Smith, C. W., Vasquez, B. J., \& Leamon, R. J. 2008, JGR, 113, A01106

He, J., Marsch, E., Tu, C., Yao, S., \& Tian, H. 2011, APJ, 731, 85

He, J., Tu, C., Marsch, E., \& Yao, S. 2012a, APJL, 745, L8

He, J., Tu, C., Marsch, E., \& Yao, S. 2012b, APJ, 749, 86

Hellinger, P., Trávníček, P. M., Kasper, J. C., \& Lazarus, A. J. 2006, GRL, 33, L09101

Horbury, T. S., Forman, M., \& Oughton, S. 2008, Phys. Rev. Lett. 101, 175005

Horbury, T. S., Matteini, L., \& Stansby, D. 2018, MNRAS, 478, 1980

Horbury, T. S., O’Brien, H., Carrasco Blazquez, I. et al. 2020, A\&A, 642, A9

Horbury, T. S., Woolley, T., Laker, R., et al. 2020, APJS, 246(2), 45

Howes, G. G., \& Quataert, E. 2010, APJL, 709, L49

Hudson, P. D. 1970, Planet. Space Sci., 18, 1611

Iroshnikov, P. S. 1963, Astronomicheskii Zhurnal 40, 742

Jagarlamudi, V. K., Alexandrova, O., Berčič, L. et al. 2020, ApJ, 897, 118

Kahler, S. W., Crooker, N. U., \& Gosling, J. T. 1996, JGR, 775101(A11), 24373

Kasper, J. C., Bale, S. D., Belcher, J. W., et al. 2019, Nature, 576(7786), 228

Kasper, J. C., Lazarus, A. J., \& Gary, S. P. 2002, GRL, 29, 1839

Kasper, J. C., Lazarus, A. J., \& Gary, S. P. 2008, PRL, 101, 261103

Kasper, J. C., Maruca, B. A., Stevens, M. L., \& Zaslavsky, A. 2013, PRL, 110, 091102
Klein, K. G., Alterman, B. L., Stevens, M. L., Vech, D., \& Kasper, J. C. 2018 , PRL, 120, 205102

Klein, K. G., Howes, G. G., TenBarge, J. M., \& Podesta, J. J. 2014, APJ, 785, 138

Klein, K. G., Martinović, M., Stansby, D., \& Horbury, T. S. 2019, APJ, 887, 234

Ko Y.-K., Roberts D. A., \& Lepri S. T., 2018, ApJ, 864, 139

Kolmogorov, A. N. 1941, Akademiia Nauk SSSR Doklady, 30, 301

Kolmogorov, A. N. 1941, Akademiia Nauk SSSR
Kraichnan, R. H. 1965, Physics of Fluids, 8, 1385

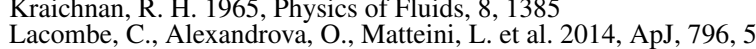

Lacombe, C., Alexandrova, O., Matteini, L. et al. 2014, ApJ, 796,
Landi, S., Hellinger, P., \& Velli, M. 2006, GRL, 33(14), L14101

Lavraud, B. et al. 2021, A\&A, this special issue, submitted

Leamon, R. J., Smith, C. W., Ness, N. F. et al. 1998, JGR, 103, A3, 4775

Lopez, R. E., \& Freeman, J. W. 1986, JGR, 91, 1701

Louarn, P. et al. 2021, A\&A, this special issue, submitted

Maksimovic, M., Bale, S. D., Chust, T. et al. 2020, A\&A 642, A12

Malara, F., Primavera, L. \& Veltri, P. 2000, Phys. Plasma, 7, 2866

Mariani, F., Bavassano, B., Villante, U., \& Ness, N.F. 1973, JGR, 78, 8011

Mariani, F., Bavassano, B., Villante, U., \& Ness, N.F. 1973, JGR, 78, 8011

Marsch, E., \& Tu, C.-Y. 1990, JGR, 95,8122

Maruca, B. A., Kasper, J. C., \& Gary, S. P. 2012, APJ 748, 137

Matteini, L., Landi, S., Hellinger, P. et al. 2007, GRL, 34, L20105

Matteini, L., Landi, S., Del Zanna, L. et al. 2010, GRL, 37, L20101

Matteini, L., Horbury, T. S., Neugebauer, M., Goldstein, B. E. 2014, GRL, 41, 259

Matteini, L., Stansby, D., Horbury, T. S., \& Chen, C. H. K. 2018, ApJ, 869, L32

Matthaeus, W. H., Goldstein, M. L., \& Smith, C. 1982, PRL, 48, 1256

Matthaeus W. H., \& Goldstein M. L., 1986, PRL, 57, 495

Matthaeus, W. H., Elliott, H. A., \& McComas, D. J. 2006, JGR, 111, A10103

McCracken, K.G., \& Ness, N.F. 1966, JGR, 71, 3315

McManus, M. D., Bowen, T. A., Mallet, A., et al. 2020, APJS, 246(2), 67

Mozer, F. S., Agapitov, O. V., Bale, S. D., et al. 2020, APJS, 246(2), 68

Müller, D., St. Cyr, O. C., Zouganelis, I., et al. 2020, A\&A, 642, A1

Narita, Y., Kleindienst, G., \& Glassmeier, K.-H. 2009, Ann. Geophys., 27, 3967

Neugebauer, M. 1981, Fundam. Cosmic Phys. 7, 131

Neugebauer, M. 1981, Fundam. Cosmic Phys. 7, 131

Neugebauer, M., Ruzmaikin, A., \& McComas, D. J. 1997, AIP Conference Proceedings, 385,41

Neugebauer, M. 2012, APJ, 750, 502012

Orlove, S. T., Smith, C. W., Vazquez, B., Schwadron, N. A. 2013, ApJ, 774, 15

Owen, C. J., Bruno, R., Livi, S. et al. 2020, A\&A 642, A16

Panasenco, O., \& Velli, M. 2013, in AIP Conf. Proc. 1539, Solar Wind 13, ed. G. P. Zank et al. (Melville, NY: AIP), 50

Panasenco, O., Martin, S. F., Velli, M., et al. 2013, Sol. Phys., 287, 391

Panasenco, O., Velli, M., \& Panasenco, A. 2019, ApJ, 873, 25

Panasenco, O., Velli, M., D'Amicis, R., et al. 2020, ApJS, 246, 54

Paschmann G., Sonnerup, B. U., Papamastorakis, I. et al. 1979, Nature, 282, 243

Perrone, D., Stansby, D., Horbury, T. S., \& Matteini, L. 2019, MNRAS, 488(2), 2380

Perrone, D., D'Amicis, R., De Marco, R. et al. 2020, A\&A, 633, A166

Podesta, J. J., \& Gary, S. P. 2011, APJ, 734, 15

Primavera, L., Malara, F., Servidio, S. et al. 2019, ApJ, 880, 156

Roberts, D.A., Klein, L.W., Goldstein, M.L., Matthaeus, W.H. 1987, JGR, 92 11021

Roberts, D. A., Goldstein, M. L., Matthaeus, W. H., \& Ghosh, S. 1992, JGR, 97, 17115

Rochus, P., Auchère, F., Berghmans, D. et al. 2020, A\&A, 642, A8

Schrijver, C. J., \& De Rosa, M. L. 2003, Sol. Phys., 212, 165

Sheeley, N. R., Martin, S. F., Panasenco, O., et al. 2013, ApJ, 772, 88

Smith, E. J. 2001, J. Geophys. Res., 106, 15819.

Solanki, S. K., del Toto Iniesta, J. C., Woch, J. et al. 2020, A\&A, 642, A11
Sonnerup, B. U. O., \& Cahill, L. J., Jr. 1967, JGR, 72, 171

Stansby, D., Matteini, L., Horbury, T. S, et al. 2020, MNRAS, 492, 39

Subramanian, S., Madjarska, M. S., \& Doyle, J. G. 2010, A\&A, 516, A50

Telloni, D., Bruno, R., D’Amicis, R. et al. 2012, APJ, 751, 19

Telloni, D., Bruno, R., \& Trenchi, L. 2015, APJ, 805, 46

Telloni, D., \& Bruno, R. 2016, MNRASL, 463, L79

Telloni, D., Carbone, F., Bruno, R., et al. 2019, APJL, 885, L5

Telloni, D., D'Amicis, R., Bruno, R., et al. 2020, APJ, 897, 167

Tenerani, Ä., \& Velli, M. 2013, JGR, 118, 7507

Torrence, C., \& Compo, G. P. 1998, Bulletin of the American Meteorological Society, 79, 61

Tsurutani, B. T., Ho, C. M., Smith, E. J., et al. 1994, GRL, 21(21), 2267

Tsurutani, B. T., Lakhina, G. S., Sen., A. et al., 2018, JGR, 123, 2458

Tu, C.-Y., \& Marsch, E. 1990, JGR, 95, 4337

Tu, C-Y. \& Marsch, E. 1993, JGR, 98, 1257

Tu, C. Y. \& Marsch, E 1995, SSR , 73 , 1

Tu, C.-Y., Pu,Z.-Y., \& Wei F.-S. 1984, JGR, 89, 9695

Tu, C.-Y., Marsch, E., \& Thieme, K. M. 1989, JGR, 94, 11739

Verdini A., Grappin R., Pinto R., Velli M., 2012, ApJ, 750, L33

Verniero, J. L., Larson, D. E., Livi, R., et al. 2020, APJS, 248, 5
Walsh, A. P., Horbury, T. S., Maksimovic, M., et al. 2020, A\&A, 642, A5

Wang, Y.-M., Grappin, R., Robbrecht, E., et al. 2012, ApJ, 749, 182

Wang, Y.-M. 2013, ApJ, 775, L46

Wicks, R. T., Roberts, D. A., Mallet, A., et al. 2013, ApJ, 778, 177

Winterhalter, D., Smith, E. J., Burton, M. E., et al. 1994, J. Geophys. Res., 99, 6667

Woodham, L. D., Wicks, R. T., Verscharen, D., \& Owen, C. J. 2018, APJ, 856, 49

Woodham, L. D., Wicks, R. T., Verscharen, D., et al. 2019, APJL, 884, L53

Woodham, L. D., Wicks, R. T., Verscharen, D., TenBarge, J. M., \& Howes, G. G. 2021, APJ, accepted

Yamauchi, Y., Suess, S. T., \& Sakurai, T. 2002, GRL, 29(10), 1383

Zouganelis, I., De Groof, A., Walsh, A. P., et al. 2020, A\&A, 642, A3 